\title{
DECIDABILITY AND INVARIANT CLASSES FOR DEGREE STRUCTURES
}

\author{
MANUEL LERMAN AND RICHARD A. SHORE
}

\begin{abstract}
We present a decision procedure for the $\forall \exists$-theory of $\mathscr{D}\left[0, \mathbf{0}^{\prime}\right]$, the Turing degrees below $\mathbf{0}^{\prime}$. The two main ingredients are a new extension of embeddings result and a strengthening of the initial segments results below $\mathbf{0}^{\prime}$ of [Le1]. First, given any finite subuppersemilattice $U$ of $\mathscr{D}\left[\mathbf{0}, \mathbf{0}^{\prime}\right]$ with top element $\mathbf{0}^{\prime}$ and an isomorphism type $V$ of a poset extending $U$ consistently with its structure as an usl such that $V$ and $U$ have the same top element and $V$ is an end extension of $U-\left\{\mathbf{0}^{\prime}\right\}$, we construct an extension of $U$ inside $\mathscr{D}\left[\mathbf{0}, \mathbf{0}^{\prime}\right]$ isomorphic to $V$. Second, we obtain an initial segment $W$ of $\mathscr{D}\left[\mathbf{0}, \mathbf{0}^{\prime}\right]$ which is isomorphic to $U-\left\{\boldsymbol{0}^{\prime}\right\}$ such that $W \cup\left\{\boldsymbol{0}^{\prime}\right\}$ is a subusl of $\mathscr{D}$. The decision procedure follows easily from these results.

As a corollary to the $\forall \exists$-decision procedure for $\mathscr{D}$, we show that no degree a $>\mathbf{0}$ is definable by any $\exists \forall$-formula of degree theory. As a start on restricting the formulas which could possibly define the various jump classes we classify the generalized jump classes which are invariant for any $\forall$ or $\exists$-formula. The analysis again uses the decision procedure for the $\forall \exists$-theory of $\mathscr{D}$. A similar analysis is carried out for the high/low hierarchy using the decision procedure for the $\forall \exists$-theory of $\mathscr{D}\left[\mathbf{0}, \mathbf{0}^{\prime}\right]$. (A jump class $\mathscr{C}$ is $\sigma$-invariant if $\sigma(\mathbf{a})$ holds for every $\mathbf{a}$ in $\mathscr{C}$.)
\end{abstract}

Introduction. This paper presents some new results dealing with decidability and definability within $\operatorname{Th}(\mathscr{D})$, the elementary theory of the poset of degrees of unsolvability. In the area of decidability, we show that $\forall \exists \cap \operatorname{Th}\left(\mathscr{D}\left[0,0^{\prime}\right]\right)$, the $\forall \exists$ theory of the degrees below $\mathbf{0}^{\prime}$, is decidable. Lachlan [La] has shown that $\mathbf{T h}(\mathscr{D})$ is undecidable, and Epstein [E] and Lerman [Le1] have obtained a similar result for $\operatorname{Th}\left(\mathscr{D}\left[\mathbf{0}, \mathbf{0}^{\prime}\right]\right)$. Schmerl (see [Le1]) has pulled both of these undecidability results down to the $\forall \exists \forall$ level. In the other direction, results of Kleene and Post [KP] can be used to show that the $\exists$-theory of each of these structures is decidable, and Shore [Sh1] and Lerman [Le1] have shown that the $\forall \exists$-theory of $\mathscr{D}$ is decidable.

We present a decision procedure for the $\forall \exists$-theory of $\mathscr{D}\left[\mathbf{0}, \mathbf{0}^{\prime}\right]$. We first prove an extension of embeddings theorem which allows us to start with a finite subuppersemilattice $\mathbf{U}$ of $\mathscr{D}\left[\mathbf{0}, \mathbf{0}^{\prime}\right]$ with top element $\mathbf{0}^{\prime}$ and an isomorphism type $\mathbf{V}$ of a poset extending $\mathbf{U}$ consistently with its structure as an usl such that $\mathbf{V}$ and $\mathbf{U}$ have the same top element and $\mathbf{V}$ is an end extension of $\mathbf{U}-\left\{\mathbf{0}^{\prime}\right\}$, and then to

Received by the editors April 15, 1987 and, in revised form, August 10, 1987.

1980 Mathematics Subject Classification (1985 Revision). Primary 03D30; Secondary 03D55.

Key words and phrases. Degrees below $\mathbf{0}^{\prime}$, extension of embeddings, initial segments, $\forall \exists$-theory, invariant jump classes, definability.

Research of the first author was partially supported by National Science Foundation grants MPS 83-018849 and DMS 85-21843.

Research of the second author was partially supported by National Science Foundation grant DMS 86-01048. 
find an extension of $\mathbf{U}$ inside $\mathscr{D}\left[\mathbf{0}, \mathbf{0}^{\prime}\right]$ isomorphic to $\mathbf{V}$. We next indicate how to modify the construction of finite initial segments below $\mathbf{0}^{\prime}$ in [Le1, Chapter XII] to obtain an initial segment $\mathbf{W}$ of $\mathscr{D}\left[\mathbf{0}, \mathbf{0}^{\prime}\right]$ which is isomorphic to $\mathbf{U}-\left\{\mathbf{0}^{\prime}\right\}$ such that $\mathbf{W} \cup\left\{\mathbf{0}^{\prime}\right\}$ is a subusl of $\mathscr{D}$. The decision procedure follows easily from these results.

We also consider the question of the definability of individual degrees and certain classes of degrees within $\mathscr{D}$, trying to place lower bounds on the complexity of any such definition. As a corollary to the $\forall \exists$-decision procedure we show (Corollary 2.8) that no degree $\mathbf{a}>\mathbf{0}$ is definable by any $\exists \forall$-formula of degree theory. The general framework for tackling the definability of $\mathbf{0}^{\prime}$ is to consider it as a special case of the problem of defining the classes of the generalized high/low hierarchy. (Note that as $\mathbf{0}^{\prime}$ is the smallest element of the class $\mathbf{G H}_{\mathbf{0}}=\left\{\mathbf{d}: d \geq \mathbf{0}^{\prime}\right\}, \mathbf{0}^{\prime}$ is definable iff $\mathbf{G H}_{\mathbf{0}}$ is definable.) We say that a hierarchy class $\mathscr{C}$ is $\sigma$-invariant if $\sigma(\mathbf{a})$ holds for every a in $\mathscr{C}$. Hence $\mathscr{C}$ is definable if there is a formula $\sigma$ (in the language of posets) with one free variable such that $\mathscr{C}$ is $\sigma$-invariant and $\overline{\mathscr{C}}$ is $\neg \sigma$-invariant. In this vein we classify the possible invariant classes for $\forall$-formulas and for $\exists$-formulas. The analysis again uses the decision procedure for the $\forall \exists$-theory of $\mathscr{D}$. A similar analysis is carried out for the high/low hierarchy using the decision procedure for the $\forall \exists$-theory of $\mathscr{D}\left[\mathbf{0}, \mathbf{0}^{\prime}\right]$.

1. Preliminaries. We use the abbreviations poset for partially ordered set and usl for upper semilattice. The usl of degrees is denoted by $\mathscr{D}$, and $\mathscr{D}[\mathbf{a}, \mathbf{b}]=$ $\{d: a \leq d \leq b\}$.

Given posets $U \subseteq V$, we call $V$ an end extension of $U$ if there do not exist $a \in U$ and $b \in V-U$ such that $b<a$. $U$ is an initial segment of $V$ if $V$ is an end extension of $U$. We call a set of degrees an initial segment if it is an initial segment of $\mathscr{D}$.

Given any degree $\mathbf{a}$, its jump, $\mathbf{a}^{\prime}$, satisfies

$$
\mathbf{a} \vee \mathbf{0}^{\prime} \leq \mathbf{a}^{\prime} \leq\left(\mathbf{a} \vee \mathbf{0}^{\prime}\right)^{\prime}
$$

The generalized high/low hierarchy measures the closeness of $\mathbf{a}^{\prime}$ to $\mathbf{a} \mathbf{V} \mathbf{0}^{\prime}$ or to $\left(\mathbf{a} \vee \mathbf{0}^{\prime}\right)^{\prime}$. This measurement is taken by computing the number of jumps, if any, which must be applied to each term in (1.1) to transform one of the inequalities into an equality. Thus the generalized high/low hierarchy $\mathscr{G}$ is defined as follows. by

We set $\mathbf{G L}_{\mathbf{0}}=\{\mathbf{0}\}$. For $n \geq 1$, define $\mathbf{G L}_{\mathbf{n}}$, the class of generalized lown degrees

$$
\mathbf{G L}_{\mathbf{n}}=\left\{\mathbf{a}: \mathbf{a}^{(n)}=\left(\mathbf{a} \vee \mathbf{0}^{\prime}\right)^{(\mathbf{n}-\mathbf{1})}\right\} .
$$

For $n \geq 0$, define $\mathbf{G H}_{\mathbf{n}}$, the class of generalized high $h_{n}$ degrees, by

$$
\mathbf{G H}_{\mathbf{n}}=\left\{\mathbf{a}: \mathbf{a}^{(n)}=\left(\mathbf{a} \vee \mathbf{0}^{\prime}\right)^{(n)}\right\} .
$$

The degrees not in any of these classes lie in GI, the class of generalized intermediate degrees, and satisfy for each $n \geq 1$,

$$
\left(\mathbf{a \vee 0 ^ { \prime }}\right)^{(n-1)}<\mathbf{a}^{(n)}<\left(\mathbf{a V 0} \mathbf{0}^{\prime}\right)^{(n)} .
$$

For any class $\mathbf{C}$ of the hierarchy, $\overline{\mathbf{C}}$ denotes the complement of $\mathbf{C}$, i.e., the set of degrees which are not in $\mathbf{C}$. For any class $\mathbf{C}$ of a high/low hierarchy, we let PC, the proper class corresponding to $\mathbf{C}$, be the set of elements which lie in $\mathbf{C}$ but not in any class properly contained in $\mathbf{C}$. Thus, e.g., $\mathbf{P G L}_{\mathbf{2}}=\mathbf{G L}_{\mathbf{2}}-\mathbf{G L}_{\mathbf{1}}$. For convenience, 
we linearly order the proper classes of the generalized high/low hierarchy under $\prec$, specifying

$$
\mathbf{P G L}_{0} \prec \mathbf{P G L}_{1} \prec \cdots \prec \mathbf{P G I} \prec \cdots \prec \mathbf{P G H}_{1} \prec \mathbf{P G H}_{0} .
$$

1.1. REMARK. The high/low hierarchy $\mathscr{H}$ has, as its classes, the classes of the generalized high/low hierarchy restricted to the degrees below $\mathbf{0}^{\prime}$. We note that the definition of the classes of the hierarchy becomes simpler in this case, as $\left(\operatorname{a\vee 0^{\prime }}\right)^{(n-1)}=0^{(n)}$ and $\left(\mathrm{a} \vee 0^{\prime}\right)^{(n)}=0^{(n+1)}$.

The ordering of the classes of the high/low hierarchy respects the ordering of the degrees (that is, $\mathbf{a} \in \mathscr{C}_{1} \& \mathbf{b} \in \mathscr{C}_{\mathbf{2}} \& \mathbf{a} \leq \mathbf{b} \Rightarrow \mathscr{E}_{\mathbf{1}} \preceq \mathscr{C}_{\mathbf{2}}$ ). This is not the case, however, for the ordering of classes of the generalized high/low hierarchy. It follows from the relativization of the join theorem of Posner and Robinson [PR] that if $\mathbf{a} \notin \mathbf{G H}_{0}$ then there is a $\mathbf{b} \in \mathbf{G L}_{\mathbf{1}}$ such that $\mathbf{b}>\mathbf{a}$. A modification, $\mathscr{G}^{*}$, of the generalized high/low hierarchy which respects the ordering of the degrees can be defined by placing the degree $\mathbf{a}$ in the proper class $\mathbf{P C}^{*}$ for the largest class $\mathbf{P C}$ under $\prec$ such that a has a predecessor in PC. There is a new class corresponding to every old class and possibly one new class corresponding to degrees which bound degrees in $\mathbf{G L}_{n}$ for all $n$, but do not bound a GI degree. (Lerman [Le2] has shown that if $\mathbf{a} \in \overline{\mathbf{G L}}_{\mathbf{2}}$, say $\mathbf{a} \in \mathbf{A}$, and $\mathbf{B} \prec \mathbf{A}$ then a bounds a degree in $\mathbf{B}$.) All known results about invariant classes for definable properties are the same for this hierarchy as for the generalized high/low hierarchy.

2. Decidability and definability. In this section, we will list the theorems needed to prove our results about decidability and definability. The decidability of the $\forall \exists$-theory of $\mathscr{D}\left[\mathbf{0}, \mathbf{0}^{\prime}\right]$ will follow as a corollary of these results, as will the nondefinability of $\mathbf{0}^{\prime}$ or indeed any nonzero degree through an $\exists \forall$-formula. These theorems will also be used in $\S 3$ to obtain results about invariant classes. Many of the theorems stated in this section were previously known, and references are provided. Proofs of the theorems new to this paper are presented in $\S \S 4$ and 5.

The language $\mathscr{L}$ which we use is the language of posets. An existential sentence of $\mathscr{L}$ asserts, when interpreted over a structure $\mathscr{M}$, that one of a finite number of partial diagrams can be embedded in $\mathscr{M}$. Thus if every finite poset can be embedded in $\mathscr{M}$, the existential sentence is true iff it is consistent with the theory of posets, a property which can be decided effectively. The following theorem indicates that this is the situation for the degree structures which we consider.

2.1. THEOREM [KP]. Any finite poset $P$ is isomorphic to a subposet $\mathscr{P}$ of $\mathbf{L}_{1} \subset \mathscr{D}$. In fact, Jockusch [J1] has shown that such an embedding exists below every $\mathbf{d} \in \overline{\mathbf{G L}}_{\mathbf{2}}$.

The next theorem is an immediate corollary of the Robinson Jump Interpolation Theorem $[\mathbf{R}]$. It is used in the next section to help in the analysis of invariant classes.

2.2. THEOREM [R]. If $\mathbf{P C}$ and $\mathbf{P D}$ are proper classes of $\mathscr{G}$ (or are both proper classes of $\mathscr{H}$ ) with $\mathbf{P C} \prec \mathbf{P D}$ and $\mathbf{c} \in \mathbf{P C}$, then there is a $\mathbf{d} \in \mathbf{P D}$ such that $\mathbf{c}<\mathbf{d}$.

The following simple fact about posets is also used in the next section. We leave its verification to the reader. 
2.3. REMARK. Let $P$ and also $Q \subseteq R$ be finite posets such that $P \simeq Q$ via an isomorphism $f$. Assume that $P$ has a greatest element $p$ and that $b \in R$ with $b>f(p)$. Let $S$ be the subposet of $R$ with universe $Q \cup\{b\}-\{f(p)\}$. Then $S \simeq P$.

The next remark describes the analysis of $\forall \exists$-sentences.

2.4. REMARK. The satisfiability of an $\forall \exists$-sentence of $\mathscr{L}$ over a structure $\mathscr{M}$ of degrees can be effectively reduced to deciding all questions of the following form [Sh1, Le1]: Let $U$ be a finite usl and let $P_{0}, \ldots, P_{n}$ be finite posets extending $U$ consistently with its usl structure. Is there then, for every realization $\mathscr{U}$ of $U$ within $\mathscr{M}$, an $i \leq n$ and an extension $\mathscr{P}_{i}$ of $\mathscr{U}$ within $\mathscr{M}$ such that $P_{i} \simeq \mathscr{P}_{i}$ ? Indeed every $\forall \exists$-sentence is equivalent to a conjunction of such statements; see Lerman [Le1, pp. 157-158] for details.

The following extension of embeddings theorem can be used together with results about initial segments to decide the $\forall \exists$-theory of $\mathscr{D}$.

2.5. THEOREM [Sh1, Le1]. Let $U$ be a finite usl with least element and let $P$ be a finite poset such that $P$ is an end extension of $U$. Let $\mathscr{U}$ be an isomorphic copy of $U$ within the degrees. Then there is an extension $\mathscr{P}$ of $\mathscr{U}$ in the degrees which is isomorphic to $\mathscr{P}$, i.e., the following diagram commutes:

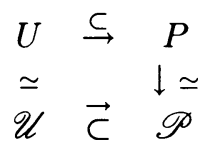

FIGURE 1

2.6. THEOREM [Le3, Le1]. Let $U$ be any finite usl with least element 0. There are then isomorphisms $f_{1}$ and $f_{2}$ of $U$ onto initial segments of $\mathscr{D}$ such that $f_{1}(x) \neq f_{2}(x)$ for all $x \in U$ other than the least element of $U$. (Note that the existence of two such embeddings follows from the existence of a single one for an usl containing two copies of $U$ disjoint except for a shared least element.)

2.7. COROllary [Sh1, Le1]. The $\forall \exists$-theory of $\mathscr{D}$ is decidable.

PROOF. By Theorems 2.5 and 2.6, an assertion about extensions of embeddings of the form mentioned in Remark 2.4 is satisfiable over the degrees iff some $P_{i}$ is an end extension of $U$.

The same results imply that there is an $\exists \forall$ definition of any degree $\mathbf{a}>\mathbf{0}$ over the degrees.

2.8. COROllary. No a $>\mathbf{0}$ is definable over $\mathscr{D}$ by an $\exists \forall$-formula.

PROOF. Let $\sigma(x)$ be an $\exists \forall$-formula of $\mathscr{L}$ with one free variable such that $\mathscr{D} \vDash \sigma(\mathbf{a})$. We show that there is a $\mathbf{b} \neq \mathbf{a}$ such that $\mathscr{D} \vDash \sigma(\mathbf{b})$.

Let $\tau$ be the sentence $\exists x \sigma(x)$. We note that $\neg \tau$ is an $\forall \exists$ sentence of $\mathscr{L}$ which is not satisfied over $\mathscr{D}$. $\neg \tau$ is equivalent to a finite conjunction of extension of embeddings statements of the type mentioned in Remark 2.4. Hence there must be a conjunct of $\neg \tau$ of this type for which no $P_{i}$ is an end extension of $U$. As, by Theorem 2.6, we can realize $U$ in $\mathscr{D}$ in different ways and fail to have an extension as required by $\neg \tau$, there is $\mathbf{a} \mathbf{b} \neq \mathbf{a}$ such that $\mathscr{D} \vDash \sigma(\mathbf{b})$ as required.

As $\mathscr{D}\left[\mathbf{0}, \mathbf{0}^{\prime}\right]$ has a greatest element but $\mathscr{D}$ does not, the $\forall \exists$-theory of $\mathscr{D}\left[\mathbf{0}, \mathbf{0}^{\prime}\right]$ differs from that of $\mathscr{D}$. New extensions of embeddings and initial segments theorems are needed to decide the $\forall \exists$-theory of $\mathscr{D}\left[\mathbf{0}, \mathbf{0}^{\prime}\right]$. They are stated below, and proved in $\S \S 4$ and 5 respectively. 
2.9. THEOREM. Let $U$ be a finite usl with least element 0 and greatest element 1 and let $P$ be a finite poset which is an end extension of $U-\{1\}$, has greatest element 1 and extends $U$ as an usl. Let $\mathscr{U}$ be an isomorphic copy of $U$ within the degrees with greatest element $\mathbf{d}$, a nonzero recursively enumerable degree. Then there is an extension $\mathscr{P}$ of $\mathscr{U}$ in the degrees which is isomorphic to $P$, i.e., the diagram of Figure 1 commutes.

2.10. THEOREM. Let $U$ be a finite usl with least element 0 and greatest element 1. Then there are isomorphisms $f_{1}$ and $f_{2}$ taking $U$ onto subusls $\mathscr{U}_{1}, \mathscr{U}_{2} \subset \mathscr{D}\left[\mathbf{0}, \mathbf{0}^{\prime}\right]$ such that $f_{i}(0)=\mathbf{0}, f_{i}(1)=\mathbf{0}^{\prime}, \mathscr{U}_{i}-\left\{\mathbf{0}^{\prime}\right\}$ is an initial segment of $\mathscr{D}$, and $f_{1}(x) \neq$ $f_{2}(x)$ for $x \in U-\{0,1\}$. (Again note that the existence of two such $f_{i}$ follows from the existence of one appropriate embedding for an usl containing two copies of $U$ disjoint except for shared least and greatest elements.)

\subsection{COROllaRY. The $\forall \exists$-theory of $\mathscr{D}\left[\mathbf{0}, \mathbf{0}^{\prime}\right]$ is decidable.}

PROOF. It suffices to decide the satisfiability of extensions of embeddings questions as specified in Remark 2.4. Let $U$ and $P_{0}, \ldots, P_{n}$ determine such a specification. By Theorem 2.10, we can choose a realization $\mathscr{U}_{1}$ of $U$ as a subusl of $\mathscr{D}\left[\mathbf{0}, \mathbf{0}^{\prime}\right]$ such that $\mathscr{U}_{1}$ has greatest element $\mathbf{0}^{\prime}$ and $\mathscr{U}_{1}-\left\{\mathbf{0}^{\prime}\right\}$ is an initial segment of $\mathscr{D}$. The above specification cannot be satisfied unless there is an $i \leq n$ such that $P_{i}$ is an usl end extension of $U-\{1\}$ with greatest element 1 (the greatest element of $U$ ). Again by Theorem 2.10, we can choose a realization $\mathscr{U}_{2}$ of $U$ which is an initial segment of $\mathscr{D}\left[\mathbf{0}, \mathbf{0}^{\prime}\right]$. The above specification cannot be satisfied unless there is also a $j \leq n$ such that $P_{j}$ is an end extension of $U$.

Suppose that such $P_{i}$ and $P_{j}$ exist. Let $\mathscr{U}$ be any realization of $U$ in $\mathscr{D}\left[\mathbf{0}, \mathbf{0}^{\prime}\right]$. If $\mathscr{U}$ has greatest element $\mathbf{0}^{\prime}$, then by Theorem $2.9, \mathscr{U}$ can be extended to a realization of $P_{i}$ in $\mathscr{D}\left[\mathbf{0}, \mathbf{0}^{\prime}\right]$. And if the greatest element of $\mathscr{U}$ is $<\mathbf{0}^{\prime}$, then again by Theorem $2.9, \mathscr{U}$ can be extended to a realization of $P_{j}$ in $\mathscr{D}\left[\mathbf{0}, \mathbf{0}^{\prime}\right]$.

2.12 Corollary. No a $>\mathbf{0}$ with $\mathbf{a}<\mathbf{0}^{\prime}$ is definable over $\mathscr{D}\left[\mathbf{0}, \mathbf{0}^{\prime}\right]$ by an $\exists \forall$-formula.

ProOF. As in the proof of Corollary 2.8 we consider an $\exists \forall$-formula $\sigma(x)$ defining a. If $\tau$ is $\exists x \sigma(x)$ then $\neg \tau$ is false in $\mathscr{D}\left[\mathbf{0}, \mathbf{0}^{\prime}\right]$ and so in the analysis of Corollary 2.11 there is either no $P_{i}$ or no $P_{j}$ as there described. In either case Theorem 2.10 gives us an embedding of $U-\{1\}$ or $U$ respectively as an appropriate initial segment of $\mathscr{D}\left[\mathbf{0}, \mathbf{0}^{\prime}\right]$ in which the element $\mathbf{b}$ corresponding to $x$ is not a. It is then clear that $\mathscr{D}\left[\mathbf{0}, \mathbf{0}^{\prime}\right] \vDash \sigma(\mathbf{b})$ for the desired contradiction.

3. Invariant classes. Let $P$ be a property definable over the poset of degrees by a formula with one free variable. The proper class PC of a hierarchy is $P$ invariant if every degree $\mathbf{a} \in \mathbf{P C}$ satisfies $P$. Given a property $P$ and a hierarchy $\mathcal{J}$, we wish to determine $\operatorname{ICL}(P, \mathcal{J})$, the union of the proper classes of $\mathscr{J}$ which are invariant for $P$. Also, given a collection $\mathscr{C}$ of properties, we wish to classify $\operatorname{ICL}(\mathscr{C}, \mathscr{J})=\{\operatorname{ICL}(P, \mathscr{J}): P \in \mathscr{C}\}$. Invariant classes have been studied for various properties of degrees. Among the known results are the following.

3.1. REMARK. Let $P_{0}$ be the property of not being a minimal degree. Yates [Y] has shown that there is a minimal degree in $\mathbf{L}_{\mathbf{1}}$ and Sasso [Sa] has shown that there is a minimal degree in $\mathbf{P L}_{\mathbf{2}}$. In the other direction, Jockusch and Posner 
[JP] have shown that every minimal degree lies in $\mathbf{G L}_{\mathbf{2}}$. Hence $\operatorname{ICL}\left(P_{0}, \mathscr{G}\right)=$ $\overline{\mathbf{G L}}_{\mathbf{2}}$. Analogous results hold for other hierarchies. Thus $\operatorname{ICL}\left(P_{0}, \mathscr{H}\right)=\overline{\mathbf{L}}_{\mathbf{2}}$ and $\operatorname{ICL}\left(P_{0}, \mathscr{G}^{*}\right)=\overline{\mathbf{G L}}_{\mathbf{2}}^{*}$.

3.2. REMARK. Let $P_{1}$ be the property of bounding a minimal degree. Jockusch [J2] has shown that every degree in $\mathbf{G H}_{\mathbf{1}}$ bounds a minimal degree, while Lerman [Le4] has shown that there are degrees in all the other proper hierarchy classes which do not bound minimal degrees. Hence $\operatorname{ICL}\left(P_{1}, \mathscr{G}\right)=\mathbf{G H}_{\mathbf{1}}, \operatorname{ICL}\left(P_{1}, \mathscr{H}\right)=$ $\mathbf{H}_{\mathbf{1}}$, and $\operatorname{ICL}\left(P_{1}, \mathscr{G}^{*}\right)=\mathbf{G H}_{\mathbf{1}}^{*}$.

3.3. REMARK. Let $P_{2}$ be the plus-cupping property, i.e., the property which asserts that a can be nontrivially joined up to every degree above it. Then Jockusch and Posner [JP] have shown that every degree in $\overline{\mathbf{G L}}_{\mathbf{2}}$ has the plus-cupping property, while the initial segments results mentioned above imply that there are degrees in $\mathbf{L}_{\mathbf{1}}$ and $\mathbf{P L}_{\mathbf{2}}$ which fail to have this property. Hence $\operatorname{ICL}\left(P_{2}, \mathscr{G}\right)=\overline{\mathbf{G L}}_{\mathbf{2}}$, $\operatorname{ICL}\left(P_{2}, \mathscr{H}\right)=\overline{\mathbf{L}}_{\mathbf{2}}$, and $\operatorname{ICL}\left(P_{2}, \mathscr{G}^{*}\right)=\overline{\mathbf{G L}}_{\mathbf{2}}^{*}$.

3.4. REMARK. Let $P_{3}$ be the Join Property, i.e., the property which asserts that every degree below $\mathbf{a}$ can be joined up to $a$ by a degree $\mathbf{b}<\mathbf{a}$. Posner and Robinson [PR] have shown that all degrees in $\mathbf{G H}_{\mathbf{1}}$ have the Join Property, while the results on minimal degrees show that the classes $\mathbf{L}_{\mathbf{1}}$ and $\mathbf{P L}_{\mathbf{2}}$ are not invariant for the Join Property. It is not known whether or not any other classes are invariant for the Join Property.

The study of invariant classes for classes of properties defined in terms of the complexity of the definitions of the properties yields a systematic approach towards trying to determine whether the degree $\mathbf{0}^{\prime}$ is the smallest element of $\mathbf{G H}_{\mathbf{0}}=$ $\left\{\mathbf{d}: \mathbf{d} \geq \mathbf{0}^{\prime}\right\}$. A positive relativizable answer to this question would allow the replacement of the known global results about the degrees with sharper results which are available for the structure of the degrees with jump. Another possible byproduct of a systematic study of invariant classes would be to determine whether or not certain hierarchy classes are definable in the language of posets, and if so, what the minimum complexity of such definitions can be. Until now the closest result to determining such a class had been a result of Shore [Sh2], proving the existence of a property $P$ such that $\mathbf{H}_{\mathbf{1}} \subseteq \operatorname{ICL}(P, \mathscr{H})$ and $\overline{\mathbf{H}_{3}} \subseteq \operatorname{ICL}(\neg P, \mathscr{H})$. He has now extended this result to show that all the jump classes from $\mathbf{L}_{\mathbf{3}}$ to $\mathbf{H}_{\mathbf{3}}$ (other than I) are definable in $\mathscr{D}\left[\mathbf{0}, \mathbf{0}^{\prime}\right][\mathbf{S h} 3]$. The complexity of the definitions is of necessity quite high as the index sets for $\mathbf{L}_{\mathbf{n}}$ and $\mathbf{H}_{\mathbf{n}}$ are $\Sigma_{n+3}$ and $\Sigma_{n+4}$ complete respectively (Schwartz). As the index set for $\mathbf{I}$ is $\Pi_{\omega+1}$ complete (Solovay) $I$ is not arithmetical and so not definable in $\mathscr{D}\left(\leq \mathbf{0}^{\prime}\right)$. (These index set results can be found in [So, Chapter XII, §4].)

Consider a property $P$ definable by a universal formula $\sigma$ with one free variable in the language of posets. Let $\mathbf{C}$ be a proper class of $\mathscr{G}$. Then $\mathbf{C}$ is $P$-invariant iff $\sigma(\mathbf{a})$ holds for all $\mathbf{a} \in \mathbf{C}$. Equivalently, $\mathbf{C}$ is not $P$-invariant if

$$
\mathscr{D} \vDash \neg \forall \mathbf{a} \in \mathbf{C} \forall \bar{x}(\sigma(\bar{x}, \mathbf{a})) .
$$

(3.1) contains an existential sentence whose truth over the degrees $\mathscr{D}$ is equivalent to the assertion that one of a finite number of partial diagrams of posets with designated element $a$ (the counterpart of $\mathbf{a} \in \mathbf{C}$ ) is embeddable in the degrees. If none of the partial diagrams is consistent with the theory of posets, then (3.1) is false, so every proper class $\mathbf{C}$ of $\mathscr{G}$ is $P$-invariant and $\operatorname{ICL}(P, \mathscr{G})=\mathscr{D}$. Suppose 
one of the partial diagrams $M$ is consistent with the theory of posets, and let $a$ be the element of $M$ corresponding to a. Let $Q$ be a finite usl extending $M$ and let $R$ be the subposet of $Q$ with universe consisting of those elements which are $\leq a$. By Theorem 2.1, there is an embedding $f$ of $R$ into $\mathscr{D}$ with $f(a) \in \mathbf{L}_{\mathbf{1}}$. By Theorem 2.2 and Remark 2.3, there is an embedding $g$ of $R$ into $\mathscr{D}$ with $g(a) \in \mathbf{C}$ as long as $\mathbf{C}$ is not $\mathbf{G L}_{\mathbf{0}}=\{\mathbf{0}\}$. As $Q$ is an end extension of $R$, it follows from Theorem 2.5 that $g(R)$ can be extended to an embedding of $Q$ into $\mathscr{D}$. Hence (3.1) holds for every choice of $\mathbf{C}$ other than $\mathbf{G L}_{\mathbf{0}}$, and $\operatorname{ICL}(P, \mathscr{G})=\varnothing$ or $\mathbf{G L}_{\mathbf{0}}$. As $\mathbf{0}$ is clearly definable by an $\forall$ formula we have proven:

3.5. THEOREM. Let $\forall$ be the class of universal formulas of $\mathscr{L}$ with one free variable. Then $\operatorname{ICL}(\forall, \mathscr{G})=\left\{\varnothing, \mathbf{G L}_{\mathbf{0}}, \mathscr{D}\right\}$.

We note that the above analysis works for $\mathscr{G}^{*}$ in place of $\mathscr{G}$ with no changes necessary. An analogous result holds for $\mathscr{H}$ as well, with the conclusion, in that case, becoming ICL $\boldsymbol{\forall}, \mathscr{H})=\left\{\varnothing, \mathbf{L}_{\mathbf{0}}, \mathbf{H}_{\mathbf{0}}, \mathscr{D}\left[\mathbf{0}, \mathbf{0}^{\prime}\right]\right\}$. As long as $\mathbf{C} \neq \mathbf{H}_{\mathbf{0}}$ we can proceed roughly as before: Form $Q^{\prime}$ from the $Q$ derived above by simply putting a new element $q$ above all those of $Q . R^{\prime}$ is the former $R$ with the new element $q$ added in. We embed $R$ as before in $L_{1}$ and, as $g(a) \neq \mathbf{0}^{\prime}$ by hypothesis, we can extend the embedding $g$ to $R^{\prime}$ by sending $q$ to $\mathbf{0}^{\prime}$. We can now apply Theorem 2.9 to $Q^{\prime}$ and $R^{\prime}$ to get an extension of $g$ embedding all of $Q^{\prime}$ in $\mathscr{D}\left[\mathbf{0}, \mathbf{0}^{\prime}\right]$. To complete the proof just note that $\mathbf{0}^{\prime}$ is definable in $\mathscr{D}\left[\mathbf{0}, \mathbf{0}^{\prime}\right]$ by an $\boldsymbol{\forall}$-formula and so $\mathbf{H}_{\mathbf{0}}$ is also invariant.

Consider a property $P$ defined by an existential formula $\sigma$ with one free variable in the language of posets. Let $\mathbf{C}$ be a proper class of $\mathscr{G}$. Then $C$ is $P$-invariant if

$$
\mathscr{D} \vDash \forall \mathbf{a} \in \mathbf{C} \exists \bar{x}(\sigma(\bar{x}, \mathbf{a})) .
$$

By Remark 2.4, (3.2) is equivalent to an assertion either stating that (3.2) is false for all $\mathbf{a}$, or one of the following form: Fix $\mathbf{a} \in \mathbf{C}$. Let $U$ be a finite usl with just a distinguished element $a$ and a least element 0 (which may be equal to $a$ if $\mathbf{a}=\mathbf{0}$ or strictly less than $a$ if $\mathbf{0}<\mathbf{a}$ ), and let $P_{1}, \ldots, P_{n}$ be finite usls extending $U$; then every isomorphic copy of $U$ within $\mathscr{D}$ in which a is interpreted as a can be extended (as a poset) within $\mathscr{D}$ to an isomorphic copy of $P_{i}$ for some $i \leq n$. There are now two cases to consider. First suppose that there is an $i \leq n$ for which $Q_{i}=\left\{b \in P_{i}: b \leq a\right\}$ has at most one element other than $a$, and that element is the least element of $U$. Then $P_{i}$ is an end extension of $U$, so by Theorem 2.5, (3.2) holds for every proper class C. Hence $\operatorname{ICL}(P, \mathscr{G})=\mathscr{D}$. Otherwise, as noted in Remark 3.1, there are minimal degrees in both $\mathbf{P L}_{\mathbf{1}}$ and $\mathbf{P L}_{\mathbf{2}}$, so (3.2) must fail if $\mathbf{C}$ is either of these classes. Suppose $\mathbf{C} \subseteq \overline{\mathbf{G L}}_{\mathbf{2}}$. By Theorem 2.1, we can embed $Q_{i}$ into the degrees $\leq \mathbf{a}$ for any specified $\mathbf{a} \in \mathbf{C}$, and by Remark 2.3, we can assume that this embedding takes $a$ to a. Theorem 2.5 now allows us to extend this embedding to one of $P_{i}$ into $\mathscr{D}$ taking $a$ to a. Hence (3.2) is seen to hold, and $\operatorname{ICL}(P, \mathscr{G})=\overline{\mathbf{G L}}_{\mathbf{2}}$. We have just proven:

3.6. THEOREM. Let $\exists$ be the class of existential formulas of $\mathscr{L}$ with one free variable. Then $\mathbf{I C L}(\exists, \mathscr{G})=\left\{\varnothing, \overline{\mathbf{G L}}_{\mathbf{0}}, \mathscr{D}, \overline{\mathbf{G L}}_{2}\right\}$.

The above proof yields the identical result for $\mathscr{G}^{*}$ in place of $\mathscr{G}$. The proof must be modified, however, for $\mathscr{H}$. The first modification necessary is to use Theorem 
2.9 in place of Theorem 2.5. Theorem 2.9 can be applied except when $\mathbf{a}=\mathbf{0}^{\prime}$. In the latter case, no end extensions are possible. Hence depending on whether new degrees below a are required and whether end extensions are required, we have additional possibilities. (Recall that $H_{0}=\left\{\boldsymbol{0}^{\prime}\right\}$.)

\subsection{Theorem. $\operatorname{ICL}(\exists, \mathscr{H})=\left\{\varnothing, \mathscr{D}\left[\mathbf{0}, \mathbf{0}^{\prime}\right], \overline{\mathbf{L}}_{\mathbf{0}}, \overline{\mathbf{L}}_{\mathbf{2}}, \overline{\mathbf{H}}_{\mathbf{0}}, \overline{\mathbf{L}}_{\mathbf{0}} \cap \overline{\mathbf{H}}_{\mathbf{0}}, \overline{\mathbf{L}}_{\mathbf{2}} \cap \overline{\mathbf{H}}_{\mathbf{0}}\right\}$.}

4. Extensions of embeddings. We begin with a simple special case of our general extension of embeddings theorem that illustrates all the ideas needed for the full theorem.

4.1. THEOREM. For any r.e. nonrecursive $D$ and any $A_{0}, A_{1}<_{T} D$ with $\left.A_{0}\right|_{T} A_{1}$ there is a $C<_{T} D$ such that, for $j=0$ and $1, A_{1-j} \Varangle_{T} A_{j} \oplus C$ and $C \Varangle_{T} A_{j}$.

To construct such a $C$ we must meet the following requirements for $j=0,1, i \in$ $\omega:$

(R) $C \leq_{T} D$

$\left(R_{4 i+2 j}\right) \theta_{i}\left(A_{j}\right) \neq C$, and

$\left(R_{4 i+2 j+1}\right) \Sigma_{i}\left(A_{j} \oplus C\right) \neq A_{1-j}$

as $\theta_{i}$ and $\Sigma_{i}$ range over all partial recursive functionals. We use the usual tree structure for dealing with the requirements as in [So]. Thus we have a tree in which each node $\alpha$ of level $|\alpha|$ is associated with requirement $R_{|\alpha|}$ which for convenience we often write as $R_{\alpha}$. Similarly we write $\theta_{\alpha}, \Sigma_{\alpha}$ etc. for $\theta_{|\alpha|}, \Sigma_{|\alpha|}, \ldots$ The $\omega$ many successors of each node $\alpha$ are $\alpha^{\wedge} q$ for each possible outcome $q$ of requirement $R_{\alpha}$. The possible outcomes will be defined and ordered $\left(<_{L}\right)$ later. The nodes $\alpha$ are then ordered by $<_{L}$ lexicographically. $\left(\alpha<_{L} \beta\right.$ is also viewed as saying $\alpha$ is to the left of $\beta$.) Our trees grow upward and so when we write $\alpha \subset \beta$ we say that $\beta$ is above $\alpha$ as well as $\beta$ extends $\alpha$ (as a sequence). We will say that a node $\alpha$ of $T$ has higher priority than a node $\beta$ if $\alpha<_{L} \beta$ or $\alpha \subset \beta$.

Before describing the modules for handling single requirements we must fix our notation and approximation procedures. We generally follow the standard convention that at a stage $s$ of any enumeration or approximation procedure no numbers larger than $s$ can be considered in any way. We are given an r.e. $D>_{T} \varnothing$ and so have a uniformly recursive nested sequence $D_{s}$ of finite sets with $\bigcup D_{s}=D$. We let $d(s)=\min \left(D_{s+1}-D_{s}\right)$ be the least element enumerated at stage $s$. A more useful approximation is given by $\widehat{D}_{s}=D_{s} \uparrow d(s)$ which incorporates the hat trick of Lachlan as in [So]. As $A_{j} \leq_{T} D$ we can fix partial recursive functionals $\Phi_{j}$ such that $A_{j}=\Phi_{j}(D)$. We view any partial recursive functional such as $\Phi_{j}$ as being approximated by a uniformly recursive sequence $\Phi_{j, s}$ of finite sets of axioms of the form $\langle x, i, \sigma\rangle$ where $x \in \omega, i \in\{0,1\}$ and $\sigma$ is a binary string, the intention being, of course, that $\Phi_{j}^{X}(x)=i$, or as we usually write, $\Phi_{j}(X ; x)=i$, iff $\exists s \exists \sigma\left(\langle x, i, \sigma\rangle \in \Phi_{j, s} \wedge \sigma \subseteq X\right)$. (We frequently, as here, confuse a set $X$ with its characteristic function.) Of course, $\Phi(X ; x, s)=i$ iff $\exists \sigma\left(\langle x, i, \sigma\rangle \subset \Phi_{s} \wedge \sigma \subseteq X\right)$. We approximate $A_{j}$ by $A_{j, s} \equiv \Phi_{j, s}\left(\widehat{D}_{s}\right) \equiv$ the longest binary string $\tau$ such that $\forall x<\operatorname{lh}(\tau) \tau \forall i \in\{0,1\}\left[\tau(x)=i \Leftrightarrow \exists \sigma\left(\langle x, i, \sigma\rangle \in \Phi_{j, s} \wedge \sigma \subseteq \widehat{D}_{s}\right)\right]$. (Thus we only define $\Phi_{s}\left(\widehat{D}_{s}\right)$ on initial segments and calculate in the style of [So] only from information $<d(s)$.) Small Greek letters $\phi, \psi, \ldots$, are used to denote the uses of such 
computations from $\Phi, \Psi$, as follows:

$$
\phi\left(\widehat{D}_{s} ; x, s\right)=\mu n>x\left(\forall y \leq x \exists i \exists \sigma\left[|\sigma|<n \&\langle x, i, \sigma\rangle \in \Phi_{s} \& \sigma \subseteq \widehat{D}_{s}\right) .\right.
$$

If there is no such $n$ we say $\Phi_{s}\left(\widehat{D}_{s} ; x\right)$ is undefined and set $\phi\left(\widehat{D}_{s} ; x, s\right)=0$. (Note that we adopt the set theoretical view that a number is the set of its predecessors. Thus the use $\phi_{s}(x)$ is a number but we say that $D$ has changed on $\phi_{s}(x)$ meaning that it has changed on the set of numbers below $\phi_{s}(x)$. In the usual functional style we would say that $D$ has changed at $\phi_{s}(x)$ if its value at the number $D\left(\phi_{s}(x)\right)$ has changed.) The notation is the same for computations relative to an entire oracle $D$ or when unrestricted as to $s$, e.g.

$$
\phi(D ; x)=\mu n>x(\forall y \leq x \exists i \exists \sigma[|\sigma|<n \&\langle x, i, \sigma\rangle \in \Phi \& \sigma \subseteq D]) .
$$

We will often omit the set variable when it is clear which is intended. We may also write $\phi_{s}(x)$ for $\phi(D ; x, s)$ or $\phi\left(\widehat{D}_{s} ; x, s\right)$ when convenient. A $D$-true stage $s$ is one for which $\forall t>s(d(t)>d(s))$. There are infinitely many $D$-true stages and as usual if $s$ is one and $\Phi_{s}\left(\widehat{D}_{s} ; x\right)=i$ then $\Phi(D ; x)=i$ and so $A_{j, s}$ is an initial segment of $A_{j}$. As $\Phi_{j}(D)=A, \lim _{s}$ is $D$-true $\Phi_{s}\left(\widehat{D}_{s}\right)=A$.

We will build $C \leq_{T} D$ by enumerating a set of axioms $\Psi$ defining $\Psi(D)=C$. We use our constructed enumeration $\Psi_{s}$ of uniformly recursive sets to define $C_{s}$. $C_{s}(y)=i$ iff $\exists \sigma \subseteq \widehat{D}_{s}\left[\langle y, i, \sigma\rangle \in \Psi_{s}\right]$. To help guarantee that $\Psi(D)$ defines a set we will at each stage $s$ have for each $x$ at most one $i \in\{0,1\}$ such that $\langle x, i, \sigma\rangle \in \Psi_{s}$ for some $\sigma \subseteq \widehat{D}_{s}$. Once we have $C_{s}(y)$ defined we will have it defined (although perhaps with different values and by different axioms) at infinitely many $t \geq s$. The only other fact that we must assure to make $\Psi(s)$ well defined is that $\lim _{s \rightarrow \infty} \psi_{s}(x)<\infty$ for each $x$.

We can now describe the module for a single requirement, i.e. how it would behave if unrestricted by other requirements. (We view $\mathbb{N}$ as divided up by coding into elements $\langle\alpha, x, s\rangle$ for $\alpha$ on our tree and $x, s \in \mathbb{N}$.)

$$
|\alpha|=4 i+2 j: R_{\alpha} \text { is } \theta_{i}\left(A_{j}\right) \neq C .
$$

We will drop the $i$ from $\theta_{i}$ and the $j$ from $A_{j}$ to simplify the notation.

The general plan is to code $D$ into $C$ as far as $\theta(A)$ and $C$ agree. Thus if $\theta(A)=C$ we would be able to recover $D$ from $A$ for a contradiction. We define the length of agreement function as usual:

$$
l(\alpha, s) \equiv \mu x\left[(\forall y<x)\left(\theta_{s}\left(A_{s} ; y\right)=C_{s}(y)\right) \wedge \neg\left(\theta_{s}\left(A_{s} ; x\right)=C_{s}(x)\right)\right] .
$$

Our goal is to guarantee that if $x<l(\alpha, s)$ and the relevant computations are correct on $A$, i.e., $A_{s}\left\lceil\theta_{s}(x)=A\left\lceil\theta_{s}(x)\right.\right.$ then $\langle\alpha, x, s\rangle \in C \Leftrightarrow x \in D$, while if not, i.e. $A_{s}\left|\theta_{s}(x) \neq A\right| \theta_{s}(x)$ then $\langle\alpha, x, s\rangle \notin C$. (If $x \geq l(\alpha, s)$ or we do not deal with $\alpha$ at $s$, then we set $C(\langle\alpha, x, s\rangle)=0$ by enumerating $\langle\langle\alpha, x, s\rangle, 0, \varnothing\rangle$ in $\Psi$.) If we accomplish this goal and $\theta(A)=C$ then we could compute $D$ from $A$ for a contradiction as follows:

To compute $D(x)$ find a stage $s$ at which $x<l(\alpha, s)$ and $A_{s} \backslash \theta_{s}(x)=A\left\lceil\theta_{s}(x)\right.$. We then have $D(x)=C(\langle\alpha, x, s\rangle)=\theta(A,\langle\alpha, s, x\rangle)$. Note that all we need to compute $D$ is that $\forall x \exists s\left[x<l(\alpha, s) \wedge A_{s} \mid \theta_{s}(x)=A\left\lceil\theta_{s}(x)\right]\right.$.

The $\alpha$-module's program. At stage $s$ we enumerate into $\Psi$ axioms $\langle\langle\alpha, x, s\rangle, 0, \varnothing\rangle$ putting $\langle\alpha, x, s\rangle$ out of $C$ forever for $x \geq l(\alpha, s)$ (as well as $\langle\langle\beta, y, s\rangle, 0, \varnothing\rangle$ for nodes $\beta$ which we are not considering at $s$ ). 
We say for $x<l(\alpha, s)$ that $\alpha$ exercises control via $s$ at every $t \geq s$ over every $y=\langle\alpha, x, s\rangle$ with assumptions $\eta(\alpha, y, s)=A_{s}\left\lceil\theta_{s}(x)\right.$. $\alpha$ 's desire for $y$ at $t \geq s$, $\alpha(y, s, t)$, is $D_{t}(x)$ if its assumptions seem correct at $t$, i.e. $\eta(\alpha, y, s) \subset A_{t}$. Otherwise $\alpha(y, s, t)=0$. $\alpha$ 's final desire for $y, \alpha(y, s)$, is of course $\lim _{t \rightarrow \infty} \alpha(y, s, t)$ which is $D(x)$ if $\eta(\alpha, y, s) \subset A$ and 0 otherwise. $\alpha$ acts to guarantee that $C(y)=\alpha(y, s)$ as follows:

For each $x<l(\alpha, s)$ we set $C_{s}(\langle\alpha, x, s\rangle)=D_{s}(x)$ via an axiom encompassing everything about $D$ used to compute the assumptions with which $\alpha$ exercises control over $y=\langle\alpha, x, s\rangle$, i.e. $\left\langle\langle\alpha, x, s\rangle, D_{s}(x), D_{s} \mid \phi_{s} \theta_{s}(x)\right\rangle$ enters $\Psi$. (Note that $\left.d(s)\right\rangle$ $\phi_{s} \theta_{s}(x)>x$ by our definitions.)

We now watch for a $D$ change on $\phi_{s} \theta_{s}(x)$ for each $x<l(\alpha, s)$. (Note that as $\phi_{s} \theta_{s}(x)>x$, we get such a change if $x$ enters $D$.) Let $t_{1}$ be the least $t \geq s$ such that $D_{t} \uparrow \phi_{s} \theta_{s}(x) \neq D_{s} \uparrow \phi_{s} \theta_{s}(x)$. (As $\Phi(D)=A$ we may assume by waiting if necessary that $\Phi_{t_{1}}\left(\widehat{D}_{t_{1}}\right)$ is defined below $\theta_{s}(x)$.) See if $\Phi_{t_{1}}\left(\widehat{D}_{t_{1}}\right) \uparrow \theta_{s}(x)=A_{s} \uparrow \theta_{s}(x)$ $\left(=\Phi_{s}\left(\hat{D}_{s}\right) \uparrow \theta_{s}(x)\right)$. If not, i.e. $A$ has changed on the use for $x$, set $C_{t_{1}}(\langle\alpha, x, s\rangle)=0$ by putting $\left\langle\langle\alpha, x, s\rangle, 0, D_{t_{1}} \uparrow \phi_{t_{1}} \theta_{s}(x)\right\rangle$ into $\Psi$. If so set $C_{t_{1}}(\langle\alpha, x, s\rangle)=D_{t_{1}}(x)$ by putting $\left\langle\langle\alpha, x, s\rangle, D_{t_{1}}(x), D_{t_{1}} \uparrow \phi_{t_{1}} \theta_{s}(x)\right\rangle$ into $\Psi$. This process is repeated at each successive $t_{i+1}$ at which $D_{t_{i+1}} \uparrow \phi_{t_{1}} \theta_{s}(x) \neq D_{t_{i}} \uparrow \phi_{t_{i}} \theta_{s}(x)$. Thus $C_{t}(y)$ is defined infinitely often for $t>s$ and always as $\alpha(y, s, t)$.

Outcomes of the $\alpha$-module. For $x \geq l(\alpha, s)$ it is clear that $\Psi(D ;\langle\alpha, x, s\rangle)=0=$ $C_{t}(\langle\alpha, x, s\rangle)=C(\langle\alpha, x, s\rangle)$ for every $t \geq s$. Consider an $x<l(\alpha, s)$ for some fixed s. As $\Phi(D)=A, \phi_{t} \theta_{s}(x)$ is eventually constant and so as $t \rightarrow \infty, C_{t}(\langle\alpha, x, s\rangle)$ also stabilizes. It is also clear that if $A \uparrow \theta_{s}(x)=A_{s} \uparrow \theta_{s}(x)$ it stabilizes at $D(x)$ while otherwise it stabilizes at 0 as desired. By our argument above there must be a least $x$ such that for no $s$ do we have $x<l(\alpha, s)$ and $A_{s} \uparrow \theta_{s}(x)=A \uparrow \theta_{s}(x)$, i.e. no computation of agreement beyond $x$ is $A$ correct. Call this $x l(\alpha)$. (Note that $l(\alpha)=\liminf _{s} l(\alpha, s)$.) It together with the $A$ correct assumptions needed for the computations $<l(\alpha)$ is the outcome of module $\alpha$.

Once $D$ has stabilized on $\phi \theta(l(\alpha))$ and all the relevant computations have appeared, say at $s_{0}$, the results of $\alpha$ 's program are clear. For $s \geq s_{0},\langle\alpha, x, s\rangle \in$ $C \Leftrightarrow x \in D$ if $x<l(\alpha)$ and $\langle\alpha, x, s\rangle \notin C$ for $x \geq l(\alpha)$. (For $s<s_{0}$ the result for $\langle\alpha, x, s\rangle$ is as described above in terms of the correctness of $A_{s} \uparrow \theta_{s}(x)$.) Thus $\alpha$ forces $C^{[\alpha]}=\{z: \exists x \exists s(z=\langle\alpha, x, s\rangle \in C)\}$ to be a recursive set whose index can be calculated uniformly from $l(\alpha)$ and any $s \geq s_{0}$.

Note also that we can guess at $\alpha$ 's final outcome at $s$ by simply guessing $\langle l(\alpha, s), \bigcup\{\eta(\alpha,\langle\alpha, x, s\rangle, s) \mid x \leq l(\alpha, s)\}\rangle$. It is clear that we guess correctly at all sufficiently large $D$-true stages $s$.

$$
|\beta|=4 c+2 j+1: R_{\beta} \text { is } \Sigma_{i}\left(A_{j} \oplus C\right) \neq A_{1-j} .
$$

We again write $\Sigma$ for $\Sigma_{i}$ and for simplicity set $j=0$.

The general plan is to guarantee that if a computation from $A_{0} \oplus C$ via $\Sigma$ which agrees with $A_{1}$ is $A_{0}$-correct then it is $C$-correct as well. Thus much as before if $\Sigma\left(A_{0} \oplus C\right)=A_{1}$ we could compute $A_{1}$ from $A_{0}$ for a contradiction.

We define the length of agreement function as before:

$$
l(\beta, s)=\mu x\left[\forall y<x\left(\Sigma_{s}\left(A_{0, s} \oplus C_{s} ; y\right)=A_{1, s}(y)\right) \& \Sigma\left(A_{0, s} \oplus C_{s} ; x\right) \neq A_{1, s}(x)\right] .
$$

Our goal here is to guarantee that if $x<l(\beta, s)$ and $A_{0}\left\lceil\sigma_{s}(x)=A_{0, s}\left\lceil\sigma_{s}(x)\right.\right.$ then $C_{s} \uparrow \sigma_{s}(x)=C \uparrow \sigma_{s}(x)$ and so if $\Sigma$ actually computed $A_{1}$ from $A_{0} \oplus C$ then we 
could recognize the final computations recursively in $A_{0}$ simply by waiting for ones which are $A_{0}$ correct. As this would give us a computation of $A_{1}$ from $A_{0}$ alone we would have our desired contradiction.

The $\beta$-module's program. Suppose $y \leq \sigma_{s}(x, s)$ for some $x<l(\beta, s)$. Let $x$ be the least such. As long as $A_{0}$ does not change on the relevant use $\sigma_{s}(x)$ we wish to keep $C$ the same on $\sigma_{s}(x)$. To do this $\beta$ asserts control via $s$ over $y$ with assumptions $\eta(\beta, y, s)=A_{0}\left\lceil\sigma_{s}(x)\right.$. (Asserting control is a declaration of a desire to control $C(y)$ by putting restrictions on the axioms that are allowed to define it. $\beta$ can actually impose (or as we say exercise) this control only in certain circumstances which we must now describe.) If $C(y)$ ever changes $\beta$ exercises its control by allowing $C(y)$ to be redefined at $t>s$ only by an axiom containing the $D$-use for $\beta$ 's assumptions: $D_{t}\left\lceil\phi_{0, t}\left(\sigma_{s}(x)\right)\right.$. We allow $C(y)$ to be redefined only when these computations exist (i.e. $\phi_{0, t}\left(\sigma_{s}(x)\right)>0$ ). As $\Phi_{0}(D)=A_{0}$ this is eventually the case. When we redefine $C(y)$ at $t, \beta$ requires that, if its assumptions seem correct, i.e. $\eta(\beta, y, s) \subseteq A_{0, t}$, we set $C_{t}(y)=C_{s}(y)=\beta(y, s, t), \beta$ 's desire for $y$ at $t$. If $\beta$ 's assumptions do not appear correct $\beta(y, s, t)$ is undefined but we may still redefine $C(y)$ at $t$, although perhaps not as $C_{s}(y)$, but rather as required by some other node of possibly lower priority. The point is that, as the defining axiom must include $D_{t} \uparrow \phi_{0, t}\left(\sigma_{s}(x)\right)$, if at any $v>t \beta$ 's assumptions once again seem correct $D$ would have changed on the axiom's use and $\beta$ would once again be able to exercise its control over $y$.

Note that it is possible to have an internal conflict between control exercised by $\beta$ over $y$ via different stages $s_{1}$ and $s_{2}$. At $s_{1}$ we may have some $z \in C_{s_{1}}$ and a computation of $\Sigma\left(A_{0} \oplus C\right)$ using this fact. As time goes by we may get a change in $A_{0}$ on the relevant $\Sigma$ use which allows $z$ to be taken out of $C$ (by some lower priority requirement). Thus at some $s_{2}>s_{1}$ we may get another $\Sigma\left(A_{0} \oplus C\right)$ computation which assumes that $z \notin C$. The use $\sigma_{2}$ at $s_{2}$ may be less than that, say $\sigma_{1}$, at $s_{1}$ and so it is possible for $A_{0}$ to return to its value at $s_{1}$ on $\sigma_{1}$ while it remains the same on $\sigma_{2}$. Thus the control asserted by $\beta$ at $s_{1}$ tries to guarantee that $z \in C$ while that at $s_{2}$ tries for $z \notin C$. In such cases we give precedence to the earlier computation, i.e. the one found at $s_{1}$ and so return $z$ to $C$. Such a conflict can arise however only if $\sigma_{1} \supset \sigma_{2}$. Thus any later change in $A_{0}\left\lceil\sigma_{1}\right.$ that keeps $A_{0}\left\lceil\sigma_{2}\right.$ as it was at $s_{2}$ allows us to take $z$ out of $C$ if desired.

Outcomes of the $\beta$-module. Consider the following inductive determination of $C$ from $A_{0}$. At each stage $s$ we have already determined some part $C_{i_{s}}$ of $C$. Let $x_{s}$ be the largest $x<l(\beta, s)$ such that $A_{0, s}\left\lceil\sigma_{s}(x)=A_{0}\left\lceil\sigma_{s}(x)\right.\right.$ and such that $C_{s}\left\lceil\sigma_{s}(x)\right.$ is not incompatible with $C_{i_{s}}$. The action of the $\beta$-module now determines that $C\left|\sigma_{s}\left(x_{s}\right)=C_{s}\right| \sigma_{s}\left(x_{s}\right)$ (so $C_{i_{s+1}}=C_{i_{s}} \cup C_{s}\left\lceil\sigma_{s}\left(x_{s}\right)\right.$ ). Thus $\Sigma\left(A_{0} \oplus C\right) \backslash x_{s}=\Sigma_{s}\left(A_{0, s} \oplus C_{s}\right) \backslash x_{s}$. If such $x_{s}$ are unbounded as $s \rightarrow \infty$ we would also recursively in $A_{0}$ correctly calculate $A_{1}$ as $\bigcup \Sigma_{s}\left(A_{0, s} \oplus C_{s}\right)\left\lceil x_{s}\right.$ by this procedure. (A difference below such an $x_{s}$ would eventually become permanent and prevent the growth of $l(\beta, s)$.) As $A_{1} \Varangle_{T} A_{0}$ there is a least $x$ which we call $l(\beta)$ such that we never get an $A$-correct computation of $\Sigma_{s}\left(A_{0, s} \oplus C_{s}\right)(x)$ for which the $C$ use also agrees with the amount of $C$ already determined for $y$. (Note that $l(\beta)=\liminf _{s} l(\beta, s)$.) Thus from some point on any computation of $\Sigma_{s}\left(A_{0, s} \oplus C_{s}\right)(x)$ is $A_{0}$ incorrect. Of course we also eventually have all the $A_{0}$ and $C$ correct computations for $y<x$ via $D$ correct computations of the associated 
uses. Thus $R_{\beta}$ controls absolutely only finitely much of $C$ and imposes restrictions on other elements which require that the axiom computing $C(x)$ from $D$ must be of some minimal length. Once one knows that this required length eventually stabilizes one sees that $R_{\beta}$ has no real impact on $C$ outside the finite set on which it attempts to determine $C$. We call the value of $l(\beta)$ together with the assumptions $\eta$ about $A$ needed for these computations and the information about $C$ determined by $\beta$ the outcome of node $\beta$ (these are specified precisely in the actual construction).

We can now describe the actual construction in which we put the various requirements together. As usual we come into each node $\gamma$ with assumptions passed on from the $\delta \subset \gamma$ which we call $\gamma$ 's prerequisites. To these $\gamma$ adds its own assumptions as in the individual modules. The major concern is to guarantee that $C$ is defined, i.e. $\Psi(D)$ is total. To that end we must make sure that the axioms used to define each $C(y)$ have finite use, i.e. we must not allow infinitely many nodes $\beta$ to exercise control over $y$. The idea here is that if $C(y)$ was last (re)defined through a node $\delta$ of higher priority than $\gamma$, there is no point in $\gamma$ 's even asserting control at $s$. It might as well rely on $\delta$. On the other hand if $C(y)$ was last (re)defined through a $\delta$ of lower priority than $\gamma$, then $\gamma$ must assert control over $y$. If, however, there is no change on the assumptions of nodes of lower priority than $\gamma$, then $\gamma$ need not really do anything. Either some $\gamma^{\prime}$ of higher priority will eventually take over $y$ or we will revert to a situation in which $C(y)$ is again redefined through $\delta$ (and so in the same way that $\gamma$ desires without any action on its part). Thus $\gamma$ will exercise control over $y$ (and so its assumptions will be relevant to the axioms used to redefine $C(y)$ ) only if some change occurs on the assumptions of lower priority exercising control over $y$ at $s$.

The nodes on our priority tree $T$ are defined by induction beginning with $\varnothing$. If $\gamma \in T$ then so is $\delta=\gamma^{\wedge} q$ where $q$ is a quadruple $\left\langle l, \mu_{0}, \mu_{1}, \mu_{2}\right\rangle$ with $l \in \omega$ and $\mu_{i} \in 2^{<\omega}$. The $\gamma^{\wedge} q=\delta$ are the immediate successors of $\gamma$. We also say that $\delta$ extends $\gamma(\delta \supset \gamma), \delta$ is above $\gamma$ or $\gamma$ is below $\delta$. The $\mu_{i}$ are the prerequisites of $\delta$. We order the quadruples and then the nodes of $T$ lexicographically. (The elements of $2^{<\omega}$, the finite binary strings, are coded as natural numbers.)

During the construction we will define the nodes of our tree which are accessible at stage $s$ by an induction through the substages of stage $s$. We say that $\alpha$ is to the left of the true path if there is an $s$ such that every $\gamma$ which is accessible at a $t>s$ is either below or to the right of $\alpha . \alpha$ is on the true path if there is an $s$ such that no node to the left of $\alpha$ is accessible after $s$ and $\alpha$ is accessible infinitely. Of course, we will have to prove by induction that there are infinitely many nodes on the true path. They are necessarily compatible and together make up what we call the true path. We say that the assumptions (prerequisites) $\eta_{0}\left(\mu_{0}\right)$ and $\eta_{1}\left(\mu_{1}\right)$ of a node $\gamma$ seem correct at $s$ if $\eta_{j}\left(\mu_{j}\right) \subset A_{j, s}$. They are correct if $\eta_{j}\left(\mu_{j}\right) \subset A_{j}$. The usage is the same for $\eta_{2}\left(\mu_{2}\right)$ and $C$.

4.2. The construction, stage $s$. We begin by attempting to redefine $C_{s}(y)$ for any $y$ at which $C$ was once defined but is no longer. (Necessarily $y=\langle\alpha, x, t\rangle$ with $|\alpha| \equiv 0(\bmod 2), x<l(\alpha, t), t<s$ and $\alpha$ is accessible at $t$.) Let $\gamma$ be the requirement of highest priority and $u$ least for $\gamma$ such that $\gamma$ exercises control over $y$ via $u$ at $s$ with assumptions which seem correct at $t$. We set $C_{s}(y)=\gamma(y, u, s)$ with use large enough to keep track of the $\eta_{0}$ and $\eta_{1}$ assumptions of all the requirements which exercise control over $y$. To be precise, we enumerate in $\Psi$ the axiom $\langle y, \gamma(y, u, s), \tau\rangle$ 
where $\tau$ is the union of $D_{s} \mid \phi_{j, s}\left(\left|\eta_{j}(\delta, y, v)\right|\right)(j=0$ or 1$)$ for all $\delta$ and $v$ such that requirement $\delta$ exercises control over $y$ at $s$ via $v$ with assumptions which seem correct assuming that all such $\phi_{j, s}\left(\left|\eta_{j}(\delta, y, v)\right|\right)$ are greater than 0 , that is all the associated computations are defined. (If they are not then we cannot redefine $C(y)$ at this stage.) In this case we say that we have redefined $C(y)$ through $\gamma$. If there is no such $\gamma$, we set $C(y)$ equal to $\alpha(y, t, s)$ with the same axiom (again only assuming that all the same computations are defined). In this case we say that we have redefined $C(y)$ through $\alpha$.

Suppose we are now at the substage of stage $s$ at which we have just declared the node $\gamma$ to be accessible with prerequisites $\mu_{j}, j \leq 2$. (The node $\varnothing$ is always accessible with prerequisites $\mu_{j}=\varnothing$.) If $|\gamma|=s$ we enumerate $\langle\langle\delta, x, s\rangle, 0, \varnothing\rangle$ into $\Psi$ for all $\delta$ which were not accessible at $s$. We then terminate stage $s$ and go on to stage $s+1$. Otherwise we proceed much as in the modules for single requirements described above in accordance with the requirement $R_{\gamma}$ associated with $\gamma$.

$|\gamma|=4 i$ : We define the length of agreement function $l(\gamma, s)$ as above. $\gamma$ exercises control via $s$ over every $y=\langle\gamma, x, s\rangle$ for $x<l(\gamma, s)$ with assumptions $\eta_{0}(\gamma, y, s)=$ $\mu_{0} \cup A_{0, s}\left\lceil\theta_{i}(x, s), \eta_{1}(\gamma, y, s)=\mu_{1}\right.$ and $\eta_{2}(\gamma, y, s)=\mu_{2}$ at every stage $t \geq s$. At $t \geq s, \gamma$ 's desire for $y$ via $s, \gamma(y, s, t)$, will be $D_{t}(x)$ if $\gamma$ 's assumptions seem correct at $t$. Otherwise, $\gamma(y, s, t)=0$. ( $\gamma$ 's final desire for $y$ is the limit of $\gamma(y, s, t)$ as $t \rightarrow \infty$, that is $D(x)$ if its assumptions are correct and 0 otherwise.) For all such $y$ we enumerate $\langle y, \gamma(y, s, s), \tau\rangle$ in $\Psi$ where $\tau=D_{s} \mid \max \left\{\phi_{j}\left(\left|\eta_{j}(\gamma, y, s)\right|\right): j=0,1\right\}$ and for all $z \geq l(\gamma, s)$ we enumerate $\langle\langle\gamma, z, s\rangle, 0, \varnothing\rangle$ in $\Psi$. The outcome of the $\gamma$ module is $q=\left\langle l(\gamma, s), \mu_{0}^{\prime}, \mu_{1}^{\prime}, \mu_{2}^{\prime}\right\rangle$ where $\mu_{0}^{\prime}=\mu_{0} \cup A_{0, s}\left\lceil\theta_{i}(l(\gamma, s)-1, s), \mu_{1}^{\prime}=\mu_{1}\right.$ and $\mu_{2}^{\prime}=\mu_{2}$. We declare $\gamma^{\wedge} q$ to be accessible with prerequisites $\mu_{0}^{\prime}, \mu_{1}^{\prime}$ and $\mu_{2}^{\prime}$ and go on to the next substage.

$|\gamma|=4 i+1$ : Again we have the appropriate length of agreement function $l(\gamma, s)$. If $y<\sigma_{i}(z, s)$ for some $z<l(\gamma, s)$ let $x$ be the least such $z$. $\gamma$ then asserts control over $y$ via $s$ with assumptions $\eta_{0}(\gamma, y, s)=\mu_{0} \cup A_{0}\left\lceil\sigma_{i}(x, s), \eta_{1}(\gamma, y, s)=\mu_{1}\right.$ and $\eta_{2}(\gamma, y, s)=\mu_{2}$ if $C(y)$ was last (re)defined through some requirement of lower priority than $\gamma$. $\gamma$ exercises control over $y$ via $s$ with assumptions $\eta_{j}(\gamma, y, s)$ at $t>s$ if for $j=0$ or 1 ,

$$
D_{t}\left\lceil\phi_{j, s}\left(\mid \eta_{j}(\delta, y, u)\right) \mid\right) \neq D_{s}\left\lceil\phi_{j, s}\left(\mid \eta_{j}(\delta, y, u)\right) \mid\right)
$$

for some $\delta$ of lower priority than $\gamma$ which exercised control over $y$ via $u$ at $s$. Its desire for $y$ at such a stage $t, \gamma(y, s, t)$, is $C_{s}(y)$. We enumerate $\langle\langle\gamma, x, s\rangle, 0, \varnothing\rangle$ in $\Psi$ for all $x$. The outcome of the $\gamma$ module is $q=\left\langle l(\gamma, s), \mu_{0}^{\prime}, \mu_{1}^{\prime}, \mu_{2}^{\prime}\right\rangle$ where $\mu_{0}^{\prime}=\mu_{0} \cup A_{0, s}\left\lceil\sigma_{i}(l(\gamma, s)-1, s), \mu_{1}^{\prime}=\mu_{1}^{\prime}\right.$, and $\mu_{2}^{\prime}=\mu_{2}^{\prime} \cup C_{s}\left\lceil\sigma_{i}(l(\gamma, s)-1, s)\right.$. We declare $\gamma^{\wedge} q$ to be accessible with prerequisites $\mu_{0}^{\prime}, \mu_{1}^{\prime}$ and $\mu_{2}^{\prime}$ and go on to the next substage.

$|\gamma|=4 i+2$ and $|\gamma|=4 i+3$ are treated as in the above cases with the appropriate alphabetic changes.

Note that for every $y, C_{s}(y)$ is defined for infinitely many $s$. The point here is that once undefined no new nodes can assert control over $y$. Thus the amounts of the $A_{j}$ that must be computed by $D$ remain fixed until $C$ is redefined at $y$. As the $A_{j}$ are in fact recursive in $D$ via the given $\Phi_{j}$, the required computations from $D$ must eventually appear. When they all do, we redefine $C(y)$ according to the directions of the construction. The problem is to show that $C(y)$ is defined in the limit. 
4.3. LEMMA. If $\alpha$ is to the left of the true path and $y=\langle\alpha, x, s\rangle$ then $C(y)$ is defined.

PROOF. The only concern is for $|\alpha| \equiv 0(\bmod 2)$ and $x<l(\alpha, s)$ with $\alpha$ accessible at $s$. It suffices to prove that there are only finitely many $\gamma$ and $t$ such that $\gamma$ ever exercises control over $y$ via $t$ with assumptions $\eta_{j}(\gamma, y, t)$ : Once $D$ has reached its final values on $\phi_{j}\left(\left|\eta_{j}(\gamma, y, t)\right|\right)$ for all such $\gamma$ and $t$ and $j=0$ or $1, C(y)$ must either be defined already by a $D$ correct axiom in $\Psi$ or it will be so defined at the next stage at which it is redefined. By construction only a $\gamma$ of higher priority than $\alpha$ could ever assert control over $y$. As nodes to the left of the true path are accessible only finitely often, we need to be concerned only with nodes $\gamma$ on the true path and below $\alpha$. For the sake of a contradiction, suppose that $\delta$ is the node on the true path below $\alpha$ of lowest priority which exercises control over $y$ via infinitely many $t$. By our choice of $\delta$ there are only finitely many $\gamma$ and $t$ with $\gamma$ of lower priority than $\delta$ such that $\gamma$ exercises control over $y$ via $t$. Once $D$ has settled down on the assumptions associated with these finitely many $\gamma$ and $t, \delta$ can assert control over $y$ but never exercise such control again by definition.

4.4. LEMMA. If $\beta$ is to the left of the true path and ever exercises control over $y$ via $s$ with correct assumptions then $C(y)$ is defined.

PROOF. By Lemma 4.3 our only concern is if $|\beta| \equiv 1(\bmod 2)$. Once $\beta$ exercises control over $y$ via $s$ and $D$ has reached its final values on the initial segment needed to correctly compute its assumptions, $C(y)$ can be redefined only through a requirement of priority at least as high as $\beta$. Thus only requirements of higher priority than $\beta$ can ever assert control over $y$ after this stage. Again just by the accessibility requirements, except for nodes $\gamma$ below $\beta$ and on the true path, only finitely many requirements can ever exercise control over $y$. For the ones below $\beta$ and on the true path we argue as in Lemma 4.3 and so here too $C(y)$ is eventually defined by a $D$ correct axiom.

We now wish to show that there is a leftmost path $f$ along which the requirements are satisfied (although perhaps because of $C$ not being defined at some $y$ ). We then argue that $C(y)$ is in fact defined for every $y$.

4.5. The MAIN LEMMA. Every $\gamma$ on the true path has correct prerequisites, is accessible at all sufficiently large D-true stages, exercises control with correct assumptions over only finitely many $y$ for $|\gamma|$ odd and over $y=\langle\gamma, x, s\rangle$ for only finitely many $x$ if $|\gamma|$ is even, is responsible for determining $C$ only on a recursive set on which it guarantees that $C$ is recursive and has an immediate successor $\gamma^{\wedge} q$ on the true path. Moreover the action taken for $\gamma$ guarantees that requirement $R_{\gamma}$ is satisfied.

PROOF. We proceed by induction on $|\gamma|$. Consider a $\gamma$ on the true path with correct prerequisites. We may choose $s_{0}$ so that after stage $s_{0}$ no node to the left of $\gamma$ is ever accessible (and so no node to the left of $\gamma$ ever asserts control over any $\left.y>s_{0}\right)$. $D$ has settled down on all the uses needed to compute the prerequisites of $\gamma$ and on $C(y)$ for $y$ controlled by nodes to the left of $\gamma$ as in Lemmas 4.3 and 4.4. On all other elements $\langle\delta, x, s\rangle$ with $\delta$ to the left of $\gamma$ we know that $C_{t}(y)=0$ for all $t \geq s$. For all elements $\langle\delta, x, s\rangle$ with $\delta$ below $\gamma$ we know by induction that the final values of $C$ are given by a recursive set, say $C_{\gamma}$. We also know by induction 
the final values of $C$ on $y$ controlled with correct assumptions by nodes of higher priority than $\gamma$.

$|\gamma|=4 i$ :

4.6. Claim. If $y=\langle\gamma, x, s\rangle$, then $C(y)$ is defined for every $x$ and $s$. Moreover, if $x<l(\gamma, s)$ for some $s\rangle s_{0}$ with $\gamma$ accessible at $s, y=\langle\gamma, x, s\rangle$ and no node of higher priurity than $\gamma$ ever exercises control over $y$ with correct assumptions, then $C(y)$ is the final desire of $\gamma$ (that is, $D(x)$ if $\gamma$ 's assumptions for $y$ are correct and 0 otherwise).

PROOF. If $\gamma$ is not accessible at $s$ or $x \geq l(\gamma, s), C(y)=0$ by construction. If $y$ is ever controlled with correct assumptions by some $\delta$ of higher priority than $\gamma$ then $C(y)$ is defined by Lemma 4.4 or by induction. We therefore suppose that $\gamma$ is accessible at $s, x<l(\gamma, s)$ and that no such $\delta$ ever controls $y$ with correct assumptions. We now show that $C(y)$ is defined and is equal to the final desire of $\gamma$.

By construction, $C(y)$ can only be defined by an axiom with $D$-use at least as long as that needed to compute the desires of $\gamma$ for $y$. If $C(y)$ is redefined at a stage at which all the assumptions with which any node of higher priority than $\gamma$ exercises control over $y$ seem incorrect, then it is then defined to be $\gamma$ 's desire. As $C$ is defined at infinitely many stages, it suffices to prove that there are only finitely many nodes $\delta$ and stages $t$ such that $\delta$ exercises control over $y$ via $t$. The point here is that in such a situation $C(y)$ must eventually be defined by a $D$-correct axiom once $D$ has stabilized on all the associated assumptions. When it is so defined it must be through $\gamma$ as the assumptions for all higher priority requirements exercising control over $y$ are incorrect.

As before the nodes to the left of $\gamma$ can assert control only finitely often by accessibility considerations alone. For those below $\gamma$ we argue by contradiction as in Lemma 4.3: If $\beta$ is the node below $\gamma$ of lowest priority that exercises control over $y$ via infinitely many $t$, there is a stage after which no node of lower priority than $\beta$ can ever exercise control over $y$. After $D$ has settled down on all the assumptions associated with such exercising of control over $y$ by nodes of lower priority, $\beta$ can assert control over $y$ but never exercise it.

Suppose now that $\theta_{i}\left(A_{0}\right)=C$ (and that both are total). It is then clear that there would be unboundedly many $x$ and $s$ as described in Claim 4.6 such that the assumptions with which $\gamma$ exercises control over $y=\langle\gamma, x, s\rangle$ are correct. As we can tell recursively in $A_{0}$ if the assumptions with which $\gamma$ exercises control over any $y$ via $s>s_{0}$ are correct and the other conditions required in Claim 4.6 can be checked recursively, we could then compute $D$ recursively in $A_{0}$ for a contradiction. Thus there is a least $x$ such that $\theta_{i}\left(A_{0} ; x\right) \neq C(x)$. Let $l(\gamma)$ be this least $x$. We have now shown that requirement $R_{4 i}$ is satisfied (although it may be as far as we now know because $C(l(\gamma))$ is undefined).

If $q$ is $l(\gamma)$ followed by the correct assumptions associated with the computations for $x<l(\gamma)$, then $\gamma^{\wedge} q$ has correct prerequisites and is the leftmost successor of $\gamma$ which is accessible infinitely often. Indeed as $\gamma$ is accessible at all sufficiently large $D$-true stages by induction, so is $\gamma^{\wedge} q$. (At such stages we can only get computations with correct assumptions.)

We can now calculate the final value of $C$ on all $y=\langle\gamma, x, s\rangle$ not already determined by nodes of higher priority. Of course, if $\gamma$ is not accessible at $s$ or $x \geq l(\gamma, s)$, 
$C(y)=0$. There are only finitely many other $y$ with $s \leq s_{0}$ and so this information for them may be assumed. For $s>s_{0}$, the claim shows that $C(y)$ is the final desire of $\gamma$, i.e., $D(x)$ if $x<l(\gamma)$ and 0 otherwise. Thus $C(\langle\gamma, x, s\rangle)$ is a recursive function of $x$ and $s$ as required.

$|\gamma|=4 i+1$ :

4.7. Claim. If, for some $s>s_{0}$ with $\gamma$ accessible at $s, y=\langle\alpha, x, t\rangle\left\langle\sigma_{i}(z, s)\right.$ with $z$ the least such $<l(\gamma, s)$, no node of higher priority than $\gamma$ ever exercises control over $y$ with correct assumptions and $\gamma$ asserts control over $y$ with correct assumptions for the first time at $s$, then $C(y)=C_{s}(y)$.

PROOF. By the definition of asserting control $C(y)$ was last defined or redefined through a node, say $\gamma_{1}$, of lower priority than $\gamma$. If $D$ ever changes, say at $s_{1}$, on its use for $\gamma_{1}$ 's assumptions or indeed for those of any $\delta$ of lower priority than $\gamma$ which exercised control over $y$ at $s$, then $\gamma$ will, from then on, exercise control over $y$ via $s$ with correct assumptions which will of course seem correct at all sufficiently large stages. Once this point is reached, say at $s_{2}$, that is $D$ has settled down on the uses for the assumptions with which $\gamma$ exercises control over $y, C(y)$ can be redefined only through nodes with priority at least that of $\gamma$. Thus no node of lower priority than $\gamma$ can ever assert control over $y$ via any $v>s_{2}$. We are now in the same situation at $s_{2}$ as we were at the beginning of the previous case. As before we see that $C(y)$ is defined. When it is redefined for the last time it must be through the node with correct assumptions of highest possible priority. By hypothesis that node is $\gamma$ and $s$ is the least number via which $\gamma$ exercises control over $y$ with correct assumptions. By the rules of the construction we must redefine $C(y)$ through $\gamma$ as $C_{s}(y)$.

We may thus assume that $D$ never changes on any of the uses for assumptions of any $\delta$ of lower priority than $\gamma$ which exercises control over $y$ at $s$. In this case no node of lower priority than $\gamma$ can ever exercise control over $y$ via any $u>s$. Moreover as $C(y)$ was redefined for the last time before $s$ through one of these nodes of lower priority, it was redefined through a node $\delta$ with correct assumptions and indeed through the one such of highest priority (and least $v$ via which it asserted control) or through $\alpha$ with its final desire for $y$. We can now argue as above that $C(y)$ is defined. When we look to see how it is last redefined, we see that it must be through the same node with the same desire for $y$ as when it was last redefined before $s$, as no node of higher priority can exercise control over $y$ with correct assumptions (none with priority higher than $\gamma$ by our hypotheses and none between $\delta$ and $\gamma$ by our choice of $\delta$ ). Of course when $C(y)$ is ever redefined in this way at $u>s$, we must set $C_{u}(y)=C_{s}(y)$ as required.

Suppose now that $\Sigma_{i}\left(A_{0} \oplus C\right)=D$.

4.8. Claim. For each $z$ there is an $s>s_{0}$ such that $\gamma$ asserts control via $s$ with correct assumptions over every $y=\langle\alpha, x, t\rangle<\sigma_{i}(z)$ (with $x<l(\alpha, t)$ and $\alpha$ of lower priority than $\gamma$ ) which is not one of the finitely many elements over which higher priority nodes exercise control with correct assumptions. Moreover we can also require that $C$ is correct at $s$ on all elements $<\sigma_{i}(z)$ which are determined by nodes of higher priority than $\gamma$.

PrOOF. Consider a $D$-true stage $s>s_{0}$ at which $\gamma$ is accessible and by which $D$ has settled down on the use needed to compute both $A_{0}$ and $C$ below $\sigma_{i}(z)$. At $s$, $l(\gamma, s)>z$. If $y<\sigma_{i}(z)=\sigma_{i}(z, s)$, then of course $C_{s}(y)$ is correctly defined and so 
necessarily was last redefined through a node with correct assumptions or through $\alpha$. As no node of higher priority than $\gamma$ ever exercises control over $y$ with correct assumptions and $\gamma$ has higher priority than $\alpha$ by hypothesis, $\gamma$ would assert control over $y$ via $s$ with correct assumptions by construction. Note that as $s$ is a $D$-true stage $\gamma$ 's assumptions must be correct.

Consider now any $z$ and $s$ as guaranteed by Claim 4.8. By Claim 4.7, $C_{s} \mid$ $\sigma_{i}(z, s)=C\left\lceil\sigma_{i}(z, s)\right.$ and so by the correctness of the assumptions assured in Claim 4.8. $\Sigma_{s}\left(A_{0, s} \oplus C ; x\right)=\Sigma\left(A_{0} \oplus C ; x\right)=D(x)$. As we can recursively in $A_{0}$ decide if the assumptions with which $\gamma$ asserts control of the $y<\sigma_{i}(z, s)$ which are not controlled by nodes of higher priority are correct (and the other requirements can be checked recursively), we could (if as we are assuming $\Sigma_{i}\left(A_{0} \oplus C\right)=D$ ) compute $D$ recursively in $A_{0}$ for a contradiction. We therefore conclude that requirement $R_{4 i+1}$ is satisfied (although once again it may, as far as we now know, be because of a divergence in $C)$. Let $l(\gamma)$ be the least $x$ such that $\Sigma\left(A_{0} \oplus C ; x\right) \neq D(x)$. It is clear that at all sufficiently large $D$-true stages the outcome $q$ of $\gamma$ is $l(\gamma)$ followed by the assumptions associated with the correct computations below $l(\gamma)$ (and so the prerequisites of $\gamma^{\wedge} q$ are correct) and that $\gamma$ exercises control with correct assumptions over only finitely many $y$ 's.

\subsection{Lemma. $C$ is defined, that is $\Psi(D)$ is total.}

P.ROOF. By Lemmas 4.3, 4.4, Claim 4.6 and, in particular, by Claim 4.8, it suffices to show that, for $y=\langle\alpha, x, s\rangle$ with $\alpha$ to the right of the true path, some $\gamma$ on or to the left of the true path asserts control over $y$ with correct assumptions. Now there is a functional $\Sigma_{i}$ such that $\langle 0, D(0), \sigma\rangle \in \Sigma_{i}$ for every $\sigma$ of length $>y$. If $\gamma$ is the node on the true path of length $4 i$ then it follows from the proof of Claim 4.8 that $\gamma$ will eventually assert control over $y$ with correct assumptions if no node of higher priority ever does. In either case $C(y)$ is defined.

The following generalization of Theorem 4.1 will suffice to prove the full extension of embeddings result (Theorem 2.9).

4.10. TheOREM. Given an r.e. $D>_{T} \varnothing$ and arbitrary sets $A_{j}<D, 0 \leq j \leq$ $n$, and an $m \in \omega$ we can build $C_{k}, k \leq m$, such that $C_{k}<_{T} D ; C_{k} \Varangle_{T} A_{j} \oplus \bigoplus_{i \neq k} C_{i}$ for each $j \leq n, k \leq m$, and $A_{j} \Varangle_{T} A_{i} \oplus \bigoplus_{k \leq m} C_{k}$ if $A_{j} \Varangle_{T} A_{i}$.

PROOF. The requirements are of two types:

(0) $\theta\left(A_{j} \oplus \bigoplus_{i \neq k} C_{i}\right) \neq C_{k}$ for $j \leq n, k \leq m$,

(1) $\Sigma\left(A_{i} \oplus \bigoplus_{k \leq m} C_{k}\right) \neq A_{j}$ for $A_{j} \not A_{i}$.

The requirements of type 1 can clearly be handled exactly as were the corresponding ones in Theorem 4.1. The requirements of type 0 are handled by a combination of the procedures used for both types of requirements in Theorem 4.1. When we have a length of agreement between $\theta\left(A_{j} \oplus \bigoplus_{i \neq k} C_{i}\right)$ and $C_{k}$ we must both preserve $C_{i}$ for $i \neq k$ and code $D$ into $C_{k}$. We may view the construction as enumerating a functional $\Psi$ such that $\Psi(D)=C=\bigcup\left\{\{k\} \times C_{k}: k \leq m\right\}$. We describe the action for the node $\gamma$ for a type 0 requirement at the appropriate substage of stage $s$.

The length of agreement function $l(\gamma, s)$ is defined as usual. $\gamma$ exercises control at every $t \geq s$ via $s$ over $\langle y, k\rangle$ for every $y=\langle\gamma, x, s\rangle$ with $x<l(\gamma, s)$ with the assumptions the prerequisites of $\gamma$ extended in the appropriate component by $A_{j, s} \uparrow$ $\theta(x, s)$. Again $\gamma$ 's desire for $\langle y, k\rangle$ at $t$ is $D_{t}(x)$ if its assumptions seem correct and 
0 otherwise. As before we enumerate $\left\langle\langle y, k\rangle, D_{t}(x), \tau\right\rangle$ in $\Psi$ where $\tau$ includes all the $D$ use on the $A$ type assumptions associated with $y$. In addition, if $y<\theta(z, s)$ for some $z<l(\gamma, s)$ we let $x$ be the least such $z$. $\gamma$ now asserts control via $s$ over $\langle y, i\rangle$ for $i \neq k$ with assumptions the prerequisites of $\gamma$ extended in the appropriate component by $A_{j, s} \uparrow \theta(x, s)$ if $\langle y, i\rangle$ was last (re)defined through a requirement of lower priority than $\gamma$. $\gamma$ exercises this control at $t>s$ if $D$ has changed by $t$ on the uses computing the various $A$ type components of the assumptions with which any $\delta$ of lower priority than $\gamma$ exercised control over $\langle y, i\rangle$ at $s$. $\gamma$ 's desire for $\langle y, i\rangle$ at such a stage $t$ is $C_{i, s}(y)=C_{s}(\langle y, i\rangle)$.

As before we set $C_{k}(\langle\gamma, z, s\rangle)=0$ for all $z \geq l(\gamma, s)$.

The argument that the construction achieves its goals is like that for Theorem 4.1. The only difference is that when dealing with type 0 requirements we must marshall the arguments for both Claims 4.6 and 4.7 before assuming (for the sake of a contradiction) that $\theta\left(A_{j} \oplus \bigoplus_{i \neq k} C_{i}\right)=C_{k}$. We must then argue as in Claim 4.8 before arguing for the final contradiction that $D \leq_{T} A_{j}$.

We now show that this suffices to give our extension of embeddings result:

2.9. THEOREM. Let $U=\left\{0,1, U_{1}, \ldots, U_{n}\right\}$ be a finite usl with least element 0 and greatest element 1 . Let $P=\left\{0,1, u_{1}, \ldots, u_{n}, p_{1}, \ldots, p_{m}\right\}$ be a finite poset which is an end extension of $U-\{1\}$ and extends $U$ as an usl. Suppose also that, for $j \leq n, A_{j} \in \mathbf{a}_{\mathbf{j}}<\mathbf{d} \ni D$ (with $D$ r.e.) are such that the map sending $u_{j} \mapsto \mathbf{a}_{\mathbf{j}}$, $0 \mapsto \mathbf{0}$ and $\mathbf{1} \mapsto \mathbf{d}$ gives an usl embedding of $U$ into $\mathscr{D}[\mathbf{0}, \mathbf{d}]$. There is then an extension to $P$ of this embedding into $\mathscr{D}[\mathbf{0}, \mathbf{d}]$.

Proof. Let $C_{j}, j \leq m$, be as in Theorem 4.10. We define the extension by $p_{k} \mapsto \operatorname{deg}\left(A_{j_{k}} \oplus \bigoplus\left\{C_{i} \mid p_{i} \leq p_{k}\right\}\right)$ where $u_{j_{k}}$ is the maximal element of $U$ below $p_{k}$. The map obviously preserves order and, by Theorem 4.10 , it preserves $\Varangle$ as well.

5. Initial segments. Let $\mathscr{L}=\langle L, \leq, \bigvee, \wedge\rangle$ be a finite lattice, with $L=$ $\left\{b_{i}: i<n\right\}$, ordered so that $b_{0}$ and $b_{1}$ are, respectively, the least and greatest elements of $L$. We let $M=\left\{m_{j}: j<j_{0}\right\}$ be the set of maximal elements of $L$, i.e. $m \in M$ if $m<b_{1}$ and there is no $b \in L$ such that $m<b<b_{1}$. We wish to embed $\mathscr{L}$, as an usl, into $\mathscr{D}\left[\mathbf{0}, \mathbf{0}^{\prime}\right]$ via an embedding map $f$ so that $f\left(b_{1}\right)=\mathbf{0}^{\prime}$ and $f\left(L-\left\{b_{1}\right\}\right)$ is an initial segment of $\mathscr{D}\left[\mathbf{0}, \mathbf{0}^{\prime}\right]$. It follows from Lerman [Le1] that this can be done if $j_{0} \leq 1$, so we assume henceforth that $j_{0}>1$.

We will indicate how to modify Lerman's proof [Le1, Chapter XII] of embeddings below $\mathbf{0}^{\prime}$ to obtain the above result. The proof will use representations of lattices as lattices of equivalence relations, and we will require that our representations have properties in addition to those required by Lerman. We first recall the notion of lattice representation, using lattice tables.

Let $\theta \subseteq \mathbb{N}^{n}$ be a set of $n$-tuples of integers. Let $\alpha, \beta \in \theta$ be given such that $\alpha=\left\langle c_{0}, \ldots, c_{n-1}\right\rangle$ and $\beta=\left\langle d_{0}, \ldots, d_{n-1}\right\rangle$, and fix $j<n$. We say that $a \equiv_{j} \beta$ if $c_{j}=d_{j}$, and use $\alpha^{[j]}$ to denote $c_{j}$, the jth coordinate of $\alpha$. We say that $\theta$ is a usl table for $\mathscr{L}$ if the following properties hold:

$$
\forall \alpha, \beta \in \theta\left(\alpha \equiv_{0} \beta\right) .
$$

$$
\forall \alpha, \beta \in \theta\left(\alpha \equiv_{1} \beta \rightarrow \alpha=\beta\right)
$$




$$
\forall i, j<n\left(b_{i} \leq b_{j} \leftrightarrow \forall \alpha, \beta \in \theta\left(\alpha \equiv_{j} \beta \rightarrow \alpha \equiv_{i} \beta\right)\right)
$$

$$
\forall i, j, k<n\left(b_{i} \vee b_{j}=b_{k} \leftrightarrow \forall \alpha, \beta \in \theta\left(\alpha \equiv_{i} \beta \& a \equiv_{j} \beta \leftrightarrow \alpha \equiv_{k} \beta\right)\right) .
$$

In order to represent lattices, we work with a sequence of usl tables with certain additional properties. Thus the sequence of $n$-tuples $\left\{\theta_{i}: i \in \mathbb{N}\right\}$ is a sequential lattice table for $\mathscr{L}$ if:

$$
\forall i \in \mathbb{N}\left(\theta_{i} \text { is a finite usl table for } \mathscr{L}\right) .
$$

$$
\begin{gathered}
\forall i \in \mathbb{N}\left(\theta_{i} \subseteq \theta_{i+1}\right) . \\
\forall i, j, k<n\left(b_{i} \wedge b_{j}=b_{k} \leftrightarrow \forall r \in \mathbb{N} \forall \alpha, \beta \in \theta_{r}\right. \\
\left(\alpha \equiv_{k} \beta \leftrightarrow \exists \gamma_{0}, \ldots, \gamma_{m} \in \theta_{r+1}\right. \\
\left.\left.\left(\alpha=\gamma_{0} \equiv_{i} \gamma_{1} \equiv_{j} \gamma_{2} \equiv_{i} \cdots \equiv_{j} \gamma_{m}=\beta\right)\right)\right) .
\end{gathered}
$$

This sequential lattice table is said to be weakly homogeneous if for all $r \in \mathbb{N}$ and $\alpha_{0}, \alpha_{1}, \beta_{0}, \beta_{3} \in \theta_{r}$, if

$$
\forall i<n\left(\alpha_{0} \equiv_{i} \alpha_{i} \rightarrow \beta_{0} \equiv_{i} \beta_{3}\right)
$$

then there are $\beta_{1}, \beta_{2} \in \theta_{r+1}$ and functions $\Psi_{s}: \theta_{r} \rightarrow \theta_{r+1}$ for $s=0,1,2$ such that for all $s \leq 2$ and $\alpha, \beta \in \theta_{r}$, the following hold:

$$
\begin{gathered}
\Psi_{s}\left(\alpha_{0}\right)=\beta_{s} \& \Psi_{s}\left(\alpha_{1}\right)=\beta_{s+1} . \\
\forall i<n\left(\alpha \equiv_{i} \beta \rightarrow \Psi_{s}(\alpha) \equiv_{i} \Psi_{s}(\beta)\right) .
\end{gathered}
$$

Weakly homogeneous sequential tables for lattices were constructed by Lerman [Le3]. (A proof is also given in [Le1, Appendix B], but the definition of weak homogeneity, the proof of Lemma 2.7 and hence that of Corollary 2.8 given there are incorrect and should be replaced with the corresponding definitions and proofs in [Le3].) The tables we will use will have two additional properties. The first is that $\theta_{0}$ should contain a subset $S$ which can be used to code $\mathbf{0}^{\prime}$ into the join of any pair of sets corresponding to elements $a, b \in L$ which do not both lie below the same element of $M$. Thus we say that the weakly homogeneous sequential table $\left\{\theta_{i}: i \in \mathbb{N}\right\}$ has $S$ as a coding set if there is a one-one map $g$ from

$$
\left\{\langle i, j, k\rangle: i<j \& k \leq 1 \& b_{i} \vee b_{j}=b_{1}\right\}
$$

onto $S \subset \theta_{0}$ with the following properties:

$$
\begin{gathered}
\forall i, j\left(i<j \& b_{i} \vee b_{j}=b_{1} \rightarrow g(i, j, 0) \equiv_{i} g(i, j, 1) \& g(i, j, 0) \not \equiv_{j} g(i, j, 1)\right) . \\
\forall \alpha \in S \forall i \exists \beta \in \theta_{0}-S\left(1<i<n \rightarrow \alpha \equiv_{i} \beta\right) .
\end{gathered}
$$

The next property will allow us to work on a tree for some fixed $a \in M$ and transfer what was done uniformly to the trees for all $c \in M$. Thus we say that the weakly homogeneous sequential lattice table $\left\{\theta_{i}\right\}$ is acceptable for $M$ if for each $r>0$, there is a subset $\theta_{r}^{*}$ of $\theta_{r}$ which contains $\theta_{r-1}$ such that (5.7) holds for $\theta_{r+1}^{*}$ in place of $\theta_{r+1},(5.8)-(5.10)$ hold for $\theta_{r+1}^{*}$ in place of $\theta_{r}$, and

$$
\begin{array}{r}
\forall i<n \forall r \forall \alpha, \beta \in \theta_{r}\left(b_{i} \in M \rightarrow \exists \gamma, \delta \in \theta_{r+1}^{*}-\theta_{r}\left(\gamma \equiv_{i} \alpha \& \delta \equiv_{i} \beta \&\right.\right. \\
\left.\left.\forall m\left(\alpha \equiv_{m} \beta \rightarrow \gamma \equiv_{m} \delta\right)\right)\right) .
\end{array}
$$


(The above definition allows us, starting with $\theta_{r}$, to find interpolants for the infimum property, to do coding using $\theta_{r+1}^{*}$ and to find homogeneity interpolants for $n$-tuples of $\theta_{r+1}^{*}$ inside $\theta_{r+1}$.) Henceforth we will use sequential table for $\mathscr{L}$ to denote a weakly homogeneous sequential lattice table for $\mathscr{L}$ with coding set $S$ which is acceptable for $M$. Thus given $\left\{\theta_{i}\right\}$, we will assume that $S$ and $\left\{\theta_{i}^{*}\right\}$ are also specified.

We now indicate how to build our sequential table. We follow the presentation in [Le1, Appendix B.2] with the exception of the proofs of Lemma 2.7 and Corollary 2.8 for which we refer the reader to [Le3]. It is proved as Lemma 2.2 of [Le1, Appendix B] that every finite lattice has a finite usl table $\theta$. Fix such a $\theta \subseteq F^{n}$ where $F$ is a finite set of integers. Let $I=\left\{\left\langle i_{q}, k_{q}\right\rangle: q<j_{2}\right\}$ be a list of all ordered pairs $\langle i, k\rangle$ such that $i<k<n, b_{i}$ and $b_{k}$ are incomparable and $b_{i} \vee b_{k}=b_{1}$, and let $J=\left\{\left\langle i_{q}, k_{q}, m_{q}\right\rangle: q\left\langle j_{3}\right\}\right.$ be a list of all ordered triples $\langle i, k, m\rangle$ such that $\langle i, k\rangle \in I$ and $m<n$. Let $\left\{F_{j}: j<j_{3}+j_{2}\right\}$ be a collection of sets of integers with cardinality $|F|$ such that if $q \neq q^{\prime}$ then $F_{q} \cap F_{q^{\prime}}=0$. Fix $q\left\langle j_{2}\right.$ and let $\langle i, k\rangle=\left\langle i_{q}, k_{q}\right\rangle$. We define a lattice table $\Xi_{q}$ for $\mathscr{L}$ by fixing a one-one correspondence $f_{q}$ between $F=F_{0}$ such that $f(0)=0$ and $F_{q}$ and letting $\alpha \in \theta$ correspond to $\beta \in \Xi_{q}$ such that $\beta^{[j]}=f_{q}\left(\alpha^{[j]}\right)$. Note that $\Xi_{q}$ and $\theta$ are isomorphic. Next, if $q=j_{2}+r$, fix $\langle i, k, m\rangle=\left\langle i_{r}, k_{r}, m_{r}\right\rangle$. Fix a one-one correspondence $f_{q}$ of $F$ with $F_{q}$ and define the lattice table $\Xi_{q}$ by letting $\alpha \in \theta$ correspond to $\beta \in \Xi_{q}$ defined by

$$
\beta^{[j]}= \begin{cases}\alpha^{[j]} & \text { if } b_{j} \leq b_{m}, \\ f_{q}\left(\alpha^{[j]}\right) & \text { otherwise. }\end{cases}
$$

Again we note that $\Xi_{q}$ is isomorphic to $\theta$. Furthermore, by the Joint Embedding Lemma [Le1, Appendix B.3.15] $\theta_{0}=\bigcup\left\{\Xi_{q}: q<j_{2}+j_{3}\right\}$ is an usl table. For $q<j_{2}$, it follows from (5.3) that there are $\alpha_{q}, \beta_{q} \in \Xi_{q}$ such that $\alpha_{q} \equiv_{i_{q}} \beta_{q}$ but $\alpha_{q} \not z_{k_{q}} \beta_{q}$. We let $g\left(i_{q}, k_{q}, 0\right)=\alpha_{q}$ and $g\left(i_{q}, k_{q}, 1\right)=\beta_{q}$, and note that (5.11) and (5.12) hold.

Suppose that $\theta_{r}$ is given. By [Le1, Corollary B.2.6], there is an usl table $\theta_{r+1}^{1} \supseteq$ $\theta_{r}$ such that (5.7) is satisfied. For each $i<n$ such that $b_{i} \in M$, we manufacture an isomorphic copy of $\theta_{r+1}^{1}$ as we defined $\Xi_{q}$ in the preceding paragraph using (5.14). Since these copies are all formed using completely new sets of integers except where precise agreement with an element of $\theta_{r+1}^{1}$ is specified, we note that, by the Joint Embedding Lemma again, the union of all such copies forms an usl table for $\mathscr{L}$ which we call $\theta_{r+1}^{*}$ and this table satisfies (5.13). Now we apply Proposition 2.2 of [Le3] to obtain an extension $\theta_{r+1}$ of $\theta_{r+1}^{*}$ satisfying (5.9) and (5.10) whenever $(5.8)$ is satisfied. We have just shown:

5.1. LEMMA. Every finite lattice $\mathscr{L}$ has a weakly homogeneous sequential lattice table with coding set $S$ and which is acceptable for the set $M$ of coatoms of $\mathscr{L}$.

Fix $i<j<n$ such that $b_{i} \vee b_{j}=b_{1}$, with $b_{i}$ and $b_{j}$ incomparable and a sequential table $\theta=\bigcup\left\{\theta_{i}: i \in \mathbb{N}\right\}$ with coding set $S$. Then, $g(i, j, 0)$ and $g(i, j, 1)$ lie in $S$. Without loss of generality, we may assume that if $\alpha=g(i, j, 0)$ and $\beta=g(i, j, 1)$, then $\alpha^{[j]}<\beta^{[j]}$. We will build a set $A$ of degree $\mathbf{0}^{\prime}$ and, for each $k \in \mathbb{N}$, will let $A^{[k]}$ be the image of $b_{k}$ under the embedding map. $\mathbf{0}^{\prime}$ will be coded into $A^{[i]} \oplus A^{[j]}$ by finding the $x$ th element $z$ such that $A^{[i]}(z) \equiv_{i} g(i, j, 0)$ and either $A^{[j]}(z) \equiv_{j}$ $g(i, j, 0)$ or $A^{[j]}(z) \equiv_{j} g(i, j, 1)$ and defining $A$ so that $x \in K \leftrightarrow A^{[j]}(z) \equiv_{j} g(i, j, 1)$. 
We indicate how to modify the construction of [Le1, Chapter XII] to accomplish this. We first note the following property of our sequential table.

5.2. Lemma. Let $\alpha, \beta \in \theta$ and $i<j<n$ be given such that $b_{i} \vee b_{j}=b_{1}$. Assume that $\alpha \equiv_{i} \beta$ and $\alpha \equiv_{j} \beta$. Then $\alpha=\beta$.

Proof. By (5.4), $\alpha \equiv_{1} \beta$ so $\alpha=\beta$.

Interspersed with the requirements to make the nontrivial sets being constructed nonrecursive, are requirements to code the r.e. set $K$ of degree $\mathbf{0}^{\prime}$ into the required joins. Thus we fix an ordering $\left\{\left\langle i_{j}, k_{j}\right\rangle: j<j_{1}\right\}$ of $\left\{\langle i, k\rangle: i<j \& b_{i}\right.$ and $b_{k}$ are incomparable $\left.\& b_{i} \vee b_{k}=b_{1}\right\}$. The sth coding requirement $\left(s=p j_{1}+q\right.$ with $\left.q<j_{1}\right)$ will code whether or not $p \in K$ for the pair $\left\langle i_{q}, k_{q}\right\rangle$. We will show that this can be accomplished while avoiding unwanted coding.

Consider the requirement to code whether or not $p \in K$ for $\langle i, j\rangle=\left\langle i_{q}, j_{q}\right\rangle$, and assume that coding has been carried out correctly for $x<p$ and no other coding has been carried out for $\langle i, j\rangle$. Then the construction will assign a tree $T$ for the satisfaction of this coding requirement such that $T(\sigma) \downarrow$ for some $\sigma$ such that $\operatorname{lh}(\sigma)=$ 1. At all stages $t$ at which attempts are made to satisfy this requirement, we will have $A_{t}^{[1]}$ extend $T(\langle g(i, j, 0)\rangle)$ as long as $p \notin K_{t}$, and $A_{t}^{[1]}$ will extend $T(\langle g(i, j, 1)\rangle)$ once $p$ enters $K_{t}$. The action taken for the coding requirements is thus similar to that taken for the nonrecursive requirements. Hence the coding requirements initiate triggering sequences just as do the nonrecursiveness requirements and so introduce no new difficulties into the construction. It remains to indicate how to define splitting trees so that no unwanted coding will occur. We refer the reader to [Le1, XII.3.4] for the splitting tree construction whose modification we next discuss.

We first note that we never have to define splitting trees corresponding to $A^{[1]}$. Rather, each of the splitting trees is localized to some $b_{i} \in M$. Thus in our search for strings with certain properties which agree $\equiv_{k}$, we need only ask for this agreement for $b_{k}<b_{i}$ to build a splitting tree with the desired properties. However, branches of the splitting tree would then be candidates for $A^{[i]}$ rather than $A^{[1]}$, so we must indicate how to extend a candidate for $A^{[i]}$ to a candidate for $A^{[1]}$. (5.13) allows us to do this freely for any fixed candidate prior to applying the Extension Lemma [Le1, VII.3.7].

Consider the construction of a new level of the splitting subtree $T$ of $T^{\prime}$. We define arrays of strings $\left\{\alpha_{j, s}: j<j_{2}\right\}$ which give an approximation, increasing in $s$, to the branches we want to place on the next level of the splitting tree. For $s=0$, we begin by tacking on all branches of $T^{\prime}$ corresponding to elements of some $\theta_{m}$. By induction we can assume that each such branch has exactly one coding argument if it corresponds to an element of $S$, and no coding arguments otherwise. We now indicate how to pass from $s$ to $s+1$ without introducing any new coding specifications. Until such extensions are made each step of the construction specifies that if a search is not successful, then $A^{[i]}$ must extend some fixed string $\gamma$. By (5.12), (5.13) and induction, this condition can be extended to requiring that $A^{[1]}$ extend some $\gamma^{\prime}$ with $\beta^{\prime[i]}=\gamma$ such that $\gamma^{\prime}$ does no coding. Now consider the two ways by which extensions are made. Either we specify that, for all $j<j_{2}$, $\alpha_{j, s+1}=\alpha_{j, s} * \beta$ for some fixed $\beta$ and so again by induction, (5.12) and (5.13) (as we need worry only about $\beta^{[i]}$ ) we can arrange it so that $\beta$ does not incorporate 
any coding specifications; or the extension is made via an extendible $e$-splitting $\langle\sigma, \tau\rangle$ with the extension map $f(j)$ determining $\beta_{j}$ such that $\alpha_{j, s+1}=\alpha_{j, s} * \beta_{j}$. (We refer the reader to [Le1, p. 240, Cases 1 and 2] to see the use of the extension map, which is denoted there by $\theta$ rather than $f$.) Now $f(j)$ is used to specify a sequence of elements of $\theta$, and no element of that sequence is in $\theta_{0}$ unless $f(j)=\sigma$ or $f(j)=\tau$. Hence, by Lemma $5.1, \beta_{j}$ will have no coding specifications with the possible exception of when $\beta_{j}$ is either $\sigma$ or $\tau$. But as the splitting tree construction ignores congruences of $\sigma$ and $\tau$ except below $b_{i}$, it follows from (5.13) that $\langle\sigma, \tau\rangle$ can be replaced with $\left\langle\sigma^{\prime}, \tau^{\prime}\right\rangle$ which are $\equiv_{r}$ with $\sigma, \tau$ respectively below $b_{i}$, and which are $\equiv_{r}$ whenever $\sigma$ and $\tau$ are $\equiv_{k}$. By the way $\theta_{r}^{*}$ was placed inside $\theta_{r}$, the Extension Lemma can be applied to $\left\langle\sigma^{\prime}, \tau^{\prime}\right\rangle$ in place of $\langle\sigma, \tau\rangle$ so that the passage from $\alpha_{j, s}$ to $\alpha_{j, s+1}$ introduces no coding specifications.

The construction of [Le1, Chapter XII], when modified as above, proves Theorem 2.10 .

Slaman [Sl] has recently shown that our Theorem 2.10 does not hold for an arbitrary r.e. degree in place of $\mathbf{0}^{\prime}$; in fact, there is a nonzero r.e. degree which is not the join of two minimal degrees. In contrast, the proof that any finite lattice is isomorphic to an initial segment of the degrees below $\mathbf{0}^{\prime}$ can be generalized, replacing $\mathbf{0}^{\prime}$ with an arbitrary nonzero r.e. degree. The difference in the ability to pull these two very similar proofs down below an arbitrary nonzero r.e. degree is due to the way coding requirements are satisfied.

In the proof of Theorem 2.10, as we build a splitting subtree $T^{*}$ of a tree $T$, we combine finitely many levels of $T$ (and prune these levels) to form a single level of $T^{*}$. This is done in such a way that the bottommost level of $T$ contributing to a given level of $T^{*}$ yields a branching pair on $T^{*}$ and the only coding done on this level of $T^{*}$ comes from this bottommost level of $T$. The branching on this bottommost level allows us to change any coding decisions as $K$ dictates such changes.

If we try to carry out a similar proof below an arbitrary nonzero r.e. degree, we can no longer guarantee that the bottommost level of $T$ contributing to a given level of $T^{*}$ provides a branching pair on $T^{*}$. This is because the r.e. permitting used to construct the splitting tree will provide us with a splitting (and hence a branching) someplace, but we cannot determine the level of $T$ for which this branching will be provided. Furthermore, as we try to construct $T^{*}$, we will not know whether we will succeed in constructing $T^{*}$ to be an infinite splitting tree. Thus we may have to commit ourselves to making certain coding decisions on levels of $T$ before the next level of $T^{*}$ is formed as we may never be able to form that next level. These coding decisions may still seem correct when we erect the next splitting level of $T^{*}$ and we may not be permitted to change them. If we are later forced to change our coding decisions, we can only do so by deleting a splitting from $T^{*}$ (destroying the r.e. ness of $T^{*}$ ); otherwise, the coding is incorrect. In the $\mathbf{0}^{\prime}$ argument, a branching is available which allows us to revise the coding decision, but it may not be present below an arbitrary nonzero r.e. degree.

6. Open questions. There are many open questions related to the topics discussed in this paper. We would like to mention several which we feel are of interest.

The limits of decidability have been reached for the structures $\mathscr{D}$ and $\mathscr{D}\left[\mathbf{0}, \mathbf{0}^{\prime}\right]$ in the language $\mathscr{L}$. One might try to obtain better decidability results by expanding 
the language $\mathscr{L}$, and deciding the $\forall \exists$-theory of the structure in the expanded language. A related question is to study $\mathscr{D}$ as an usl with jump. The associated language would then have a relation symbol $<$ and a function symbol $j$ interpreted as the jump operator over the degrees.

Question 6.1. Is the $\exists$-theory of the degrees with jump decidable?

While there is no direct counterpart to Question 1 for $\mathscr{D}\left[\mathbf{0}, \mathbf{0}^{\prime}\right]$, one could instead expand the language with two-place predicates $J_{n}(a, b)$ whose interpretation is $\mathbf{a}^{(n)}=\mathbf{b}^{(n)}$ and try to decide the corresponding $\exists$-theory.

Having classified $\operatorname{ICL}(\forall, \mathscr{C})$ and $\operatorname{ICL}(\exists, \mathscr{C})$ for $\mathscr{C} \in\left\{\mathscr{G}, \mathscr{H}, \mathscr{G}^{*}\right\}$, one would next naturally turn to the next level of complexity. Let $\forall \exists$ ( $\exists \forall$ respectively) denote the $\forall \exists$-formulas ( $\exists \forall$-formulas, respectively) of $\mathscr{L}$ with one free variable.

Question 6.2. Classify ICL $(\forall \exists, \mathscr{C})$ for the above hierarchies $\mathscr{C}$.

Question 6.3. Classify $\operatorname{ICL}(\exists \forall, \mathscr{C})$ for the above hierarchies $\mathscr{C}$.

The first roadblock in classifying $\operatorname{ICL}(\exists \forall, \mathscr{G})$ is the classification of the invariant classes for the following property.

Question 6.4. Let $P$ be the property of being a minimal cover of a minimal degree. Find $\operatorname{ICL}(P, \mathscr{G})$.

A problem of particular interest at the $\forall \exists \forall$ level is the Join Property.

Question 5. Let $P$ be the Join Property. Find $\operatorname{ICL}(P, \mathscr{G})$.

\section{REFERENCES}

[E] R. L. Epstein, Initial segments of degrees below 0', Mem. Amer. Math. Soc. No. 241 (1981).

[J1] C. G. Jockusch, Jr., Degrees of generic sets, Recursion Theory: Its Generalisations and Applications (F. Drake and S. Wainer, Eds.), London Math. Soc. Lecture Notes Series, no. 45, Cambridge Univ. Press, Cambridge, 1980, pp. 110-139.

[J2] _ Simple proofs of some theorems on high degrees of unsolvability, Canad. J. Math. 29 (1970). 1072-1080.

[JP] C. G. Jockusch, Jr. and D. Posner, Double jumps of minimal degrees, J. Symbolic Logic 43 (1978), 715-724.

[KP] S. C. Kleene and E. L. Post, The uppersemilattice of degrees of recursive unsolvability, Ann. of Math. (2) 59 (1954), 379-407.

[La] A. H. Lachlan, Distributive initial segments of the degrees of unsolvability, Z. Math. Logik Grundlag. Math. 14 (1968), 457-472.

[Le1] M. Lerman, Degrees of unsolvability, Perspectives in Mathematical Logic, Springer-Verlag, Berlin, Heidelberg, New York, Tokyo, 1983, 307 pages.

[Le2] _ _ On the ordering of classes in high/low hierarchies, Recursion Theory Week (H. D. Ebbinghaus, G. H. Müller, and G. E. Sacks, Eds.), Lecture Notes in Math., vol. 1145, Springer-Verlag, Berlin, Heidelberg, 1985, pp. 260-270.

[Le3] _ Initial segments of the degrees of unsolvability, Ann. of Math. (2) 93 (1971), 365-389.

[Le4] __ Degrees which do not bound minimal degrees, Ann. Pure Appl. Logic 30 (1986), 249-276.

[PR] D. Posner and R. W. Robinson, Degrees joining to $\mathbf{0}^{\prime}$, J. Symbolic Logic 46 (1981), 714-722.

[R] R. W. Robinson, Jump restricted interpolation in the r.e. degrees, Ann. of Math. (2) 93 (1971), 586-596.

[Sa] L. P. Sasso, Jr., A minimal degree not realizing least possible jump, J. Symbolic Logic 39 (1974), 571-574.

[Sh1] R. A. Shore, On the $\forall \exists$-sentences of $\alpha$-recursion theory, Generalized Recursion Theory. II (J. Fenstad, R. Gandy, and G. Sacks, Eds.), Stud. Logic Foundations Math., no. 94, NorthHolland, Amsterdam, 1978, pp. 331-354.

[Sh2] — The theory of the degrees below 0', J. London Math. Soc. (2) 24 (1981), 1-14.

[Sh3] _ _ Defining jump classes in the degrees below 0', Proc. Amer. Math. Soc. 104 (1988), 287-292. 
[S1] T. Slaman, An r.e. degree which is not the top of a diamond, in preparation.

[So] R. I. Soare, Recurisvely enumerable sets and degrees, Springer-Verlag, Berlin, 1987.

[Y] C. E. M. Yates, Initial segments of the degrees of unsolvability. Part II: Minimal degrees, J. Symbolic Logic 35 (1970), 243-266.

Department of Mathematics, University of Connecticut, Storrs, ConNECTICUT 06268

Department of Mathematics, Cornell University, Ithaca, NeW York 14853 